\title{
The epidemiology of hepatitis C virus in Egypt: a systematic review and data synthesis
}

\author{
Yousra A Mohamoud', Ghina R Mumtaz', Suzanne Riome', DeWolfe Miller ${ }^{4}$ and Laith J Abu-Raddad ${ }^{1}, 2,3^{*}$
}

\begin{abstract}
Background: Egypt has the highest prevalence of hepatitis $\mathrm{C}$ virus (HCV) in the world, estimated nationally at $14.7 \%$. Our study's objective was to delineate the evidence on the epidemiology of HCV infection among the different population groups in Egypt, and to draw analytical inferences about the nature of HCV transmission in this country.

Methods: We conducted a systematic review of all data on HCV prevalence and incidence in Egypt following PRISMA guidelines. The main sources of data included PubMed and Embase databases. We also used a multivariate regression model to infer the temporal trend of HCV prevalence among the general population and high risk population in Egypt.

Results: We identified 150 relevant records, four of which were incidence studies. HCV incidence ranged from 0.8 to 6.8 per 1,000 person-years. Overall, HCV prevalence among pregnant women ranged between $5-15 \%$, among blood donors between 5-25\%, and among other general population groups between 0-40\%. HCV prevalence among multi-transfused patients ranged between 10-55\%, among dialysis patients between 50-90\%, and among other high risk populations between $10 \%$ and $85 \%$. HCV prevalence varied widely among other clinical populations and populations at intermediate risk. Risk factors appear to be parenteral anti-schistosomal therapy, injections, transfusions, and surgical procedures, among others. Results of our time trend analysis suggest that there is no evidence of a statistically significant decline in HCV prevalence over time in both the general population ( $p$-value: 0.215 ) and high risk population ( $p$-value: 0.426 ).

Conclusions: Egypt is confronted with an HCV disease burden of historical proportions that distinguishes this nation from others. A massive HCV epidemic at the national level must have occurred with substantial transmission still ongoing today. HCV prevention in Egypt must become a national priority. Policymakers, and public health and medical care stakeholders need to introduce and implement further prevention measures targeting the routes of HCV transmission.
\end{abstract}

Keywords: Hepatitis C Virus, Epidemiology, Prevalence, Incidence, Egypt, Systematic Review

\section{Background}

The Egyptian Demographic Health Survey (EDHS), a cross sectional survey including hepatitis $\mathrm{C}$ virus (HCV) biomarkers, was conducted in 2008 on a large nationally representative sample [1]. It estimated $\mathrm{HCV}$ prevalence among the 15-59 years age group to be $14.7 \%$ [1]. Accordingly, Egypt has the highest $\mathrm{HCV}$ prevalence in the world [2-4]. This unparalleled level of exposure

\footnotetext{
*Correspondence: lja2002@qatar-med.cornell.edu

'Infectious Disease Epidemiology Group, Weill Cornell Medical College -

Qatar, Cornell University, Qatar Foundation - Education City, Doha, Qatar

${ }^{2}$ Department of Public Health, Weill Cornell Medical College, Cornell

University, New York, New York, USA

Full list of author information is available at the end of the article
}

to this infection appears to reflect a national level epidemic. It has been postulated that the epidemic has been caused by extensive iatrogenic transmission during the era of parenteral-antischistosomal-therapy (PAT) mass-treatment campaigns [5,6]. Today, HCV infection and its complications are among the leading public health challenges in Egypt [7].

Multiple community- and facility-based studies were conducted among different population groups in Egypt over the last two decades to assess the distribution of infection in the population. These studies have immensely improved our understanding of $\mathrm{HCV}$ epidemiology in Egypt. Nevertheless, two lingering and critical questions

\section{Biomed Central}


regarding $\mathrm{HCV}$ transmission in Egypt are yet to be addressed satisfactorily:

1) Does the high HCV prevalence reflect mainly historical exposures during the PAT campaigns before 1985, with limited current infection incidence?

2) If not, to what extent is HCV transmission still ongoing, and what are the drivers, risk factors, and modes of this transmission?

The objective of our study is to attempt, at least in part, to address these two questions through a comprehensive systematic review and integrated analysis of multiple sources of data about $\mathrm{HCV}$ prevalence and incidence in Egypt. Our study examined side-by-side information collected by different methods, by different investigators, and in different populations, allowing us to corroborate hypotheses across datasets, thereby reducing the impact of potential biases that can exist in a single study, dataset, or line of evidence. Our approach also facilitated an identification of the key research, policy, and programming priorities that require further investigation and consideration.

\section{Methods}

\section{Data sources and search strategy}

We conducted a systematic review of the prevalence and incidence of $\mathrm{HCV}$ in the different population groups in Egypt following the PRISMA guidelines [8]. The PRISMA checklist can be found in Table S1, see Additional file 1. The main data sources for this investigation were: PubMed (Medline) and Embase databases. The above data sources were searched with no time or language restrictions. PubMed and Embase were searched using both $\mathrm{MeSH} /$ Emtree terms, respectively, and text terms. MeSH/ Emtree terms were exploded to cover all subheadings. Details of the search criteria for each of these databases can be found below:

PubMed: (("Hepatitis C"[Mesh] OR "Hepatitis C Antibodies"[Mesh] OR "Hepatitis C Antigens"[Mesh] OR "Hepacivirus"[Mesh] OR "Hepatitis C, chronic/ epidemiology"[Mesh] OR "Hepatitis C, chronic/etiology" [Mesh] OR "Hepatitis C, chronic/transmission"[Mesh] OR "Hepatitis C, chronic/virology"[Mesh] OR "Hepatitis C"[Text] OR "HCV"[Text]) AND ("Egypt"[Mesh] or Egypt [Text])).

Embase: (egypt.mp. or exp Egypt/) and (exp hepatitis C/ or exp Hepatitis C virus/ or hepatitis C.mp. or HCV.mp. or hepacivirus.mp.).

\section{Ethics statement}

Our study did not need an ethics committee approval or written informed consent because it relies entirely on published data.

\section{Study selection}

The results of the searches were imported to a reference manager, Endnote, where duplicate publications were identified and excluded. The remaining unique and potentially relevant records were then imported into Microsoft Excel where screening for relevance and eligibility took place. The titles and abstracts of all records retrieved were screened for relevance independently by two of the authors (YM and SR). Screening for relevance was conducted in two stages: 1) Stage 1 involved screening all titles and abstracts to exclude all non-relevant articles; 2) Stage 2 involved retrieving and screening the full-text of all articles deemed relevant after the initial abstract screening, to further exclude any remaining non-eligible articles. Inconsistencies between reviewers were discussed and sorted out by consensus.

A publication was considered eligible for inclusion in the review if it had data on at least one of the following outcomes of interest: 1) prevalence of $\mathrm{HCV}$ as detected by HCV antibodies; and 2) incidence of HCV as detected by $\mathrm{HCV}$ antibodies. Only studies reporting primary data were included. Reviews of literature were excluded, but all data reported were checked and compared to the results of our search. Any additional study identified in the excluded review and not retrieved by our search was identified and added to our review. Case reports and case series were excluded. All other study designs were eligible for inclusion. Distinction was made between the number of "reports" (actual publications i.e. papers, conference abstracts etc.) and the number of "studies" (actual study and research project). Multiple reports of the same study were identified as duplicates and counted as one study.

Eligible studies were then categorized into two types: prevalence studies and incidence studies. Any article reporting both the prevalence and incidence of $\mathrm{HCV}$ was counted as two studies, one for incidence and one for prevalence. Results were then pooled into one list containing all eligible and unique studies.

\section{Data extraction and population classification}

The following data were then extracted from each eligible study included in the review: author, year of data collection, year of publication, city, study site, study design, sampling technique, population (blood donors, barbers, health care workers, injecting drug users (IDUs) etc.), socio-demographic characteristics of the population (sex, age, rural vs. urban etc.), sample size, and prevalence and/or incidence of HCV. Although our search criteria did not specifically target publications reporting $\mathrm{HCV}$ RNA prevalence or risk factors in Egypt, we extracted this information from eligible publications when available. Risk factors were extracted only if they were statistically 
significant in a multivariate regression analysis within a study that was found relevant according to our search criteria.

Data on the above mentioned indicators were extracted from included records and entered into a computerized database on Microsoft Excel. Extracted data were then classified and analyzed on the basis of the study population's risk of acquiring $\mathrm{HCV}$. The four defined major population risk groups are:

1) Populations at direct or high risk: this group includes IDUs, multi-transfused patients such as hemophiliacs and thalassemics, dialysis patients, and viral hepatitis patients, among others.

2) Populations at indirect or intermediate risk: this group includes familial contacts of HCV patients i.e. their children, spouses, and other household contacts; select practitioners of professions at risk of $\mathrm{HCV}$ such as dentists, healthcare workers and barbers; populations with potential IDU exposures including: prisoners and HIV patients; and populations with health facility/injecting exposures such as diabetic patients and hospitalized populations.

3) General population groups (populations which are not at an elevated risk of HCV exposure): pregnant women, blood donors, children, rural populations, army recruits or fire brigade personnel, outpatient clinic attendees, populations defined in case-control studies as healthy populations (controls), among other groups categorized together as "other general population" groups.

4) Special clinical population groups such as Hodgkin's lymphoma (HL) patients, lichen planus (LP), and liver disease patients, among others. This category includes patients with specific diseases that require clinical care, and thus can be exposed to HCV in medical care facilities, though at variable levels of risk that is difficult to categorize among any of the above mentioned population groups.

Within any specific category of the above population groups, considerable heterogeneity and different subgroup trends may exist.

In order to create prevalence figures, $\mathrm{HCV}$ prevalence measures within each of the population groups above were stratified into two strata: pre-2001 and post-2001, based on the year in which the study was conducted. The year 2001 was chosen as the cut-off year, as this was the year in which the Egyptian Ministry of Health initiated broad infection control programs in the country [9].

\section{Time trend analysis}

We conducted a time trend analysis investigating the change in $\mathrm{HCV}$ prevalence among the general population and high risk population in Egypt with respect to time. We started by conducting univariate linear regression analyses examining the change in $\mathrm{HCV}$ prevalence over time in each of the general population subgroups separately. A similar analysis was conducted in each of the high risk population subgroups. To avoid systematic biases in any one subgroup and to increase the statistical power of the time trend analysis, we also used a multivariate linear regression model estimating the temporal trend in $\mathrm{HCV}$ prevalence, while adjusting for the different subgroups in each population grouping. Two such models were performed: one for the general population and the other for the high-risk population. Mean HCV prevalence was modeled using a model that includes as predictors: time (in years) and the different general population subgroups (or high risk population subgroups).

The general population regression model stipulates that:

$$
\begin{aligned}
\text { Mean } & \text { HCV Prevalence }=\beta_{0}+\beta_{1} * \text { Time } \\
& +\beta_{2} * \text { Antenatal clinic attendees } \\
& +\beta_{3} * \text { Blood donors }+\beta_{4} * \text { Rural village residents } \\
& +\beta_{5} * \text { Children }+\beta_{6} * \text { Healthy populations } \\
& +\beta_{7} * \text { Army recruits }+\beta_{8} * \text { Other }
\end{aligned}
$$

Similarly, the high risk population regression model stipulates that:

$$
\begin{aligned}
\text { Mean } & \text { HCV Prevalence }=\beta_{0}+\beta_{1} * \text { Time } \\
& +\beta_{2} * \text { Multi }- \text { transfused patients } \\
& +\beta_{3} * \text { Schistosomiasis patients } \\
& +\beta_{4} * \text { Thalassemic patients } \\
& +\beta_{5} * \text { Viral hepatitis patients }
\end{aligned}
$$

In both models, $\beta i$ are the parameters of the statistical model and Time and sub-population names are the indicator variables.

Data used for these analyses were extracted from the eligible studies included in this review. In the presence of both an overall HCV prevalence measure as well as stratified prevalence measures, we included only the overall prevalence measure. Including both overall and stratified prevalence measures from the same study would have given more weight to those studies, relative to others, thereby biasing our results. The year of data collection was estimated for studies missing this variable. This was done by conducting a correlation analysis between the year of data collection and the year of publication for studies having data on both, followed by a paired t-test to estimate the mean difference between them. We then applied this difference to the year of publication to estimate the year of data collection when missing. Midpoints were calculated and used for studies conducted over a number of years. The statistical analysis was 
conducted using STATA version 11 (STATA corporation, College Station, Texas).

\section{Results}

\section{Search results}

The study selection process is described in Figure 1, as adapted from the PRISMA 2009 flow diagram [8]. The number of records retrieved through both PubMed and Embase was 1,146 as of August 1, 2012, out of which 445 were excluded as duplicates. After assessing all documents according to their titles and abstracts, the full-text of 183 records were retrieved for screening in addition to 11 papers identified through references of reviews. Of those, 150 records were found eligible for inclusion in the present article. Only one relevant record was identified outside the PubMed and Embase search; the EDHS [1]. No single report has reported both a relevant incidence measure and a relevant prevalence measure.

\section{HCV incidence}

Five incidence measures were identified through our search, reported in four incidence reports (Table 1). All studies were conducted in rural areas of Egypt among village residents, pregnant women, and children [10-13]. Overall high HCV incidence rates were observed in these studies ranging from 0.8 to 6.8 per 1,000 person-years.

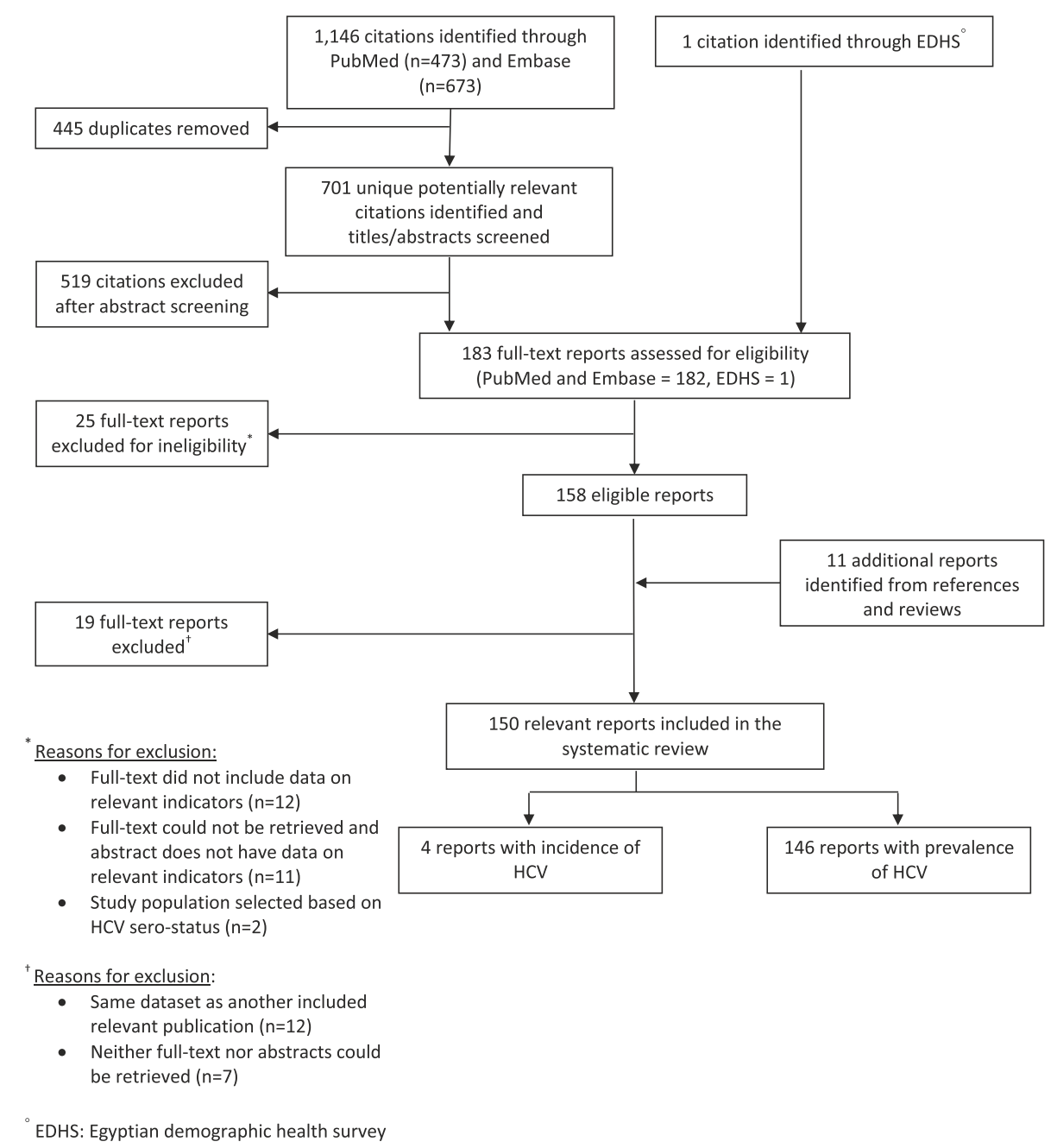

Figure 1 Flow of article selection for the HCV prevalence and incidence in Egypt search. This chart, adapted from the PRISMA 2009 flow diagram, displays the flow of article selection for the HCV incidence and prevalence in Egypt search of scientific databases, namely PubMed and Embase. 
Table 1 Studies reporting hepatitis C virus incidence in Egypt

\begin{tabular}{|c|c|c|c|c|c|}
\hline Citation & Year & Location & Study population & Sample size & $\begin{array}{r}\text { Incidence (per } 1,000 \\
\text { person-years) }\end{array}$ \\
\hline Mohamed,05 [14] & $1997-2000$ & Qalubyia, Lower Egypt & Village residents & 2,463 & 6.8 \\
\hline Mohamed,05 [14] & $1997-2000$ & Assuit, Upper Egypt & Village residents & 4,275 & 0.8 \\
\hline Saleh,08 [15] & 1997-2006 & Menoufia, Lower Egypt & Pregnant women & 2,177 & 5.2 \\
\hline Mostafa,10 [16] & $2001-2003$ & Menoufia, Lower Egypt & Village residents & 3,580 & 2 \\
\hline Saleh,10 [17] & $2000-2006$ & Menoufia, Lower Egypt & Children of 3 villages with high prevalence of HCV & 2,852 & 2.7 \\
\hline
\end{tabular}

\section{Prevalence of HCV in the general population}

Sixty-nine studies reported HCV prevalence in the general population. Details are shown in Table 2. HCV prevalence among the general population of Egypt is documented to be very high. The 2008 EDHS measured HCV prevalence to be $14.7 \%$ among a nationally representative sample of 11,126 Egyptians aged 15-59 years old [1]. The diverse HCV studies conducted among different general population subgroups, regardless of design or methodology, consistently report a very high HCV prevalence, as high as $41 \%$ in some studies [10]. Overall, the prevalence appears to increase dramatically with age with the highest rates observed among populations aged greater than 40 years.

A number of studies were conducted among blood donors. A higher prevalence is observed among paid blood donors and family replacement blood donors compared to voluntary donors [10-12]. Male blood donors had a higher prevalence than their female counterparts [13]. Blood donors from rural areas had a higher prevalence than those from urban areas [38].

Multiple studies were conducted among village residents in high HCV prevalence areas (Table 2). The overall prevalence in rural areas averaged about $20 \%$, higher than the national average. A study conducted in Kalama, a village in the Nile Delta, reported HCV prevalence of $40 \%$ among village residents [60]. Similar to blood donor studies, village residents were shown to have a higher prevalence among males compared to females [62,81], and a marked growth in prevalence with age $[56,58,59]$. A study conducted in 1997, among 3,993 residents of a village in the Nile Delta region, observed prevalence rates in children, ages $0-19$, ranging between 7 and $9.9 \%$. This rate increased to $27.6 \%$ in those $20-39$ years and more than doubled to $56.7 \%$ among village residents greater than 40 years of age [82].

High HCV prevalence was also observed among pregnant women and children in Egypt. Recent studies conducted among pregnant women reported a prevalence of about $8 \%$ in Assuit [27] and Benha [28], and as high as $15.8 \%$ in rural villages of the Nile Delta [26]. Studies conducted among rural school children reported an average prevalence of about $7 \%$ [53,66,68,69], while the average prevalence in children attending outpatient clinics was found to be approximately $4 \%[18,19,21,22]$. High prevalence was also observed among select subgroups such as tourism workers [70], army recruits [53,79] and fire brigade personnel [34].

Figure 2A depicts the range of prevalence within each subgroup in studies conducted pre- and post-2001. Among blood donors, studies appear to cluster at lower HCV prevalence levels post- 2001 infection control programs, compared to pre-2001. However, no distinct pattern can be observed within each of the other subgroups.

\section{Prevalence of HCV among populations at direct or high risk of exposure}

We classified populations at direct or high risk of HCV exposure into six subcategories: viral hepatitis patients, multitransfused patients, thalassemia patients, schistosmiasis patients, patients on hemodialysis and IDUs. Table 3 lists all the studies in each sub-category and the reported prevalence measures.

Among patients diagnosed with acute viral hepatitis, $\mathrm{HCV}$ prevalence ranged from as low as $4.3 \%$ [89] to as high as $78.7 \%$ [85]. Once more, we observed a higher prevalence among studies conducted in rural populations versus urban populations. A recent study conducted in 2010 reported an HCV prevalence of $8.7 \%$ among children with viral hepatitis [87].

The majority of studies in multi-transfused and thalassemia patients were conducted among children. High $\mathrm{HCV}$ prevalence rates were observed with averages of about $42 \%$ among multi-transfused children and about $58 \%$ among children with thalassemia. Multiple studies were also conducted among hemodialysis patients (mostly adults). Very high HCV prevalence was found in both adult populations and children on hemodialysis.

There were six studies that investigated $\mathrm{HCV}$ prevalence among schistosomiasis patients. Of these, only two explicitly mentioned previous PAT exposure. However, from the studies context, and given the high $\mathrm{HCV}$ prevalence across all of these studies, exposure to previous PAT campaigns seems to be implicitly assumed. Accordingly, we chose to classify these six studies in one subgroup: schistosomiasis patients, and not separate them into two categories based on previous PAT exposure. 
Table 2 Studies reporting hepatitis C virus prevalence among the general population in Egypt

\begin{tabular}{|c|c|c|c|c|c|c|c|}
\hline Citation & Year & Location & Sampling & Population characteristic & Sample size & Sero-prevalence & RNA prevalence \\
\hline \multicolumn{8}{|c|}{ Outpatient clinic attendees } \\
\hline Khalifa,93 [18] & 1990-1 & Cairo city, Cairo & CS & Children & 84 & $0.0 \%$ & $\mathrm{~N} / \mathrm{A}$ \\
\hline El-Nanawy,95 [19] & N/A & Alexandria city, Alexandria & CS & Children & 110 & $11.8 \%$ & N/A \\
\hline Miras,02 [20] & N/A & Tanta, Gharbia, Lower Egypt & CS & $\begin{array}{l}\text { In/out-patient children living in HCV } \\
\text { endemic region }\end{array}$ & 105 & $0.0 \%$ & $\mathrm{~N} / \mathrm{A}$ \\
\hline El-Raziky,07 [21] & 2004 & Cairo city, Cairo & CS & Children 1-9 yrs old & 1,042 & $1.4 \%$ & $0.5 \%$ \\
\hline Kandil,07 [22] & $2004-6$ & Cairo city, Cairo & CS & Healthy children & 20 & $5.0 \%$ & $\mathrm{~N} / \mathrm{A}$ \\
\hline \multicolumn{8}{|c|}{ Antenatal clinic attendees } \\
\hline Hassan,93 [23] & N/A & N/A & CS & & 1,536 & $4.3 \%$ & N/A \\
\hline Agha,98 [24] & $1996-7$ & Mansoura, Dakahlia, Lower Egypt & CS & & 767 & $13.7 \%$ & $23.7 \%$ \\
\hline Kassem,00 [25] & 1996 & Alexandria city, Alexandria & CS & & 100 & $19.0 \%$ & $14.0 \%$ \\
\hline Stoszek,05 [26] & $1997-03$ & 3 rural villages in Nile River Delta, Lower Egypt & CS & & 2,587 & $15.8 \%$ & $10.8 \%$ \\
\hline Zahran,10 [27] & $2008-9$ & Assuit, Upper Egypt & CS & & 500 & $8.0 \%$ & $7.4 \%$ \\
\hline Abdulqawi,10 [28] & $2003-8$ & Benha, Qalubiya, Lower Egypt & CS & & 1,224 & $8.6 \%$ & $6.8 \%$ \\
\hline Abo Elmagd,11 [29] & N/A & N/A & CS & 20-40 year old mothers & 61 & $13.0 \%$ & N/A \\
\hline \multicolumn{8}{|l|}{ Blood donors } \\
\hline Kamel,92 [30] & 1992 & Cairo city, Cairo & CS & Male university students, 20-27 years & 2,164 & $9.7 \%$ & N/A \\
\hline El-Zayadi,92 [31] & N/A & N/A & CS & & 76 & $5.2 \%$ & N/A \\
\hline Darwish,92 [32] & N/A & N/A & CS & & 90 & $14.4 \%$ & N/A \\
\hline Darwish,93 [33] & 1992 & Cairo city, Cairo & CS & & 163 & $13.6 \%$ & N/A \\
\hline El-Ahmady,94 [10] & N/A & N/A & CS & Paid blood donors & 99 & $35.4 \%$ & $\mathrm{~N} / \mathrm{A}$ \\
\hline Quinti,95 [34] & $1992-4$ & Alexandria city, Alexandria & CS & & 283 & $20.8 \%$ & N/A \\
\hline Bassily,95 [35] & N/A & Cairo city, Cairo & CS & & 188 & $26.6 \%$ & N/A \\
\hline El Gohary,95 [36] & $1990-2$ & Suez city, Suez and Ismailia, Lower Egypt & CS & & 1,187 & $14.5 \%$ & N/A \\
\hline Attia,96 [37] & N/A & Cairo city, Cairo & CS & & 156 & $21.8 \%$ & $\mathrm{~N} / \mathrm{A}$ \\
\hline Arthur,97 [38] & 1993 & 24 Governorates & CS & & 2,644 & $24.8 \%$ & N/A \\
\hline El-Zayadi,97 [39] & N/A & N/A & CS & & 180 & $9.4 \%$ & $\mathrm{~N} / \mathrm{A}$ \\
\hline Gad,01 [40] & 1998 & Ismailia, Lower Egypt & CS & & 20 & $20.0 \%$ & $\mathrm{~N} / \mathrm{A}$ \\
\hline Tanaka,04 [41] & 1999 & 13 governorates, Upper and Lower Egypt & CS & & 3,608 & $8.8 \%$ & $6.2 \%$ \\
\hline Hashish,05 [42] & N/A & Alexandria city, Alexandria & CS & & 95 & $23.2 \%$ & N/A \\
\hline El-Gilany,06 [43] & $2002-3$ & Mansoura, Dakahlia, Lower Egypt & CS & Student voluntary blood donors & 2,157 & $2.7 \%$ & N/A \\
\hline Agha,06 [44] & N/A & N/A & CS & & 2,400 & $8.0 \%$ & N/A \\
\hline El Damaty,07 [45] & 2001 & Cairo city, Cairo & CS & & 2,845 & $7.6 \%$ & $\mathrm{~N} / \mathrm{A}$ \\
\hline
\end{tabular}


Table 2 Studies reporting hepatitis C virus prevalence among the general population in Egypt (Continued)

\begin{tabular}{|c|c|c|c|c|c|c|c|}
\hline El-Zayadi,08 [46] & 2005 & 26 governorates & Random & All blood donors & 760 & $5.0 \%$ & $\mathrm{~N} / \mathrm{A}$ \\
\hline El-Zayadi,08 [46] & 2005 & 26 governorates & & Female blood donors & 124 & $6.5 \%$ & N/A \\
\hline El-Zayadi,08 [46] & 2005 & 26 governorates & & Male blood donors & 636 & $4.7 \%$ & N/A \\
\hline Ismail,09 [47] & $2000-7$ & Mansoura, Dakahlia, Lower Egypt & CS & & 55,922 & $12.0 \%$ & N/A \\
\hline Ashour,09 [48] & 2006-8 & 8 governorates & CS & & 515,758 & $4.8 \%$ & N/A \\
\hline Elkareh,09 [11] & 2008 & Menoufia, Lower Egypt & CS & Family replacement blood donors & 4,709 & $12.7 \%$ & N/A \\
\hline Elkareh,09 [11] & 2008 & Menoufia, Lower Egypt & CS & Blood donors & 3,569 & $6.3 \%$ & $\mathrm{~N} / \mathrm{A}$ \\
\hline Elkareh,09 [11] & 2008 & Menoufia, Lower Egypt & CS & Family replacement blood donors & 8,705 & $14.6 \%$ & N/A \\
\hline Elkareh,09 [11] & 2008 & Menoufia, Lower Egypt & CS & Blood donors & 414 & $8.7 \%$ & N/A \\
\hline Rushdy,09 [13] & $2006-7$ & Suez Canal area & CS & All blood donors & 9,150 & $5.6 \%$ & N/A \\
\hline Rushdy,09 [13] & $2006-7$ & Suez Canal area & & Male blood donors & 7,155 & $2.9 \%$ & $\mathrm{~N} / \mathrm{A}$ \\
\hline Rushdy,09 [13] & $2006-7$ & Suez Canal area & & Female blood donors & 1,995 & $1.7 \%$ & N/A \\
\hline Eita,09 [12] & $2005-8$ & Dakhilia, Lower Egypt & CS & Voluntary blood donors & 73,431 & $4.6 \%$ & N/A \\
\hline Eita,09 [12] & 2005-8 & Dakhilia, Lower Egypt & CS & Family blood donors & 113,504 & $5.5 \%$ & N/A \\
\hline Khattab,10 [36] & $2000-8$ & Minya, Lower Egypt & CS & & 211,772 & $9.0 \%$ & N/A \\
\hline Radwan,10 [49] & 2009 & N/A & CS & & 27,537 & $4.0 \%$ & N/A \\
\hline Wasfi,11 [50] & $2007-8$ & Alexandria city, Alexandria & CS & & 3,420 & $3.5 \%$ & N/A \\
\hline Awadalla,11 [51] & N/A & Cairo city, Cairo & CS & & 1,000 & $16.8 \%$ & N/A \\
\hline Farawela,12 [52] & 2010-1 & Cairo city, Cairo & CS & & 100 & $5 \%$ & N/A \\
\hline \multicolumn{8}{|c|}{ Rural village residents } \\
\hline Abdel-Wahab,94 [53] & 1992 & Menoufia, Lower Egypt & CS & & 270 & $18.1 \%$ & N/A \\
\hline Kamel,94 [54] & 1992 & Sada, Kafr El Sheikh, Lower Egypt & $\begin{array}{l}\text { All village } \\
\text { residents }\end{array}$ & & 1,259 & $15.9 \%$ & N/A \\
\hline El Gohary,95 [36] & $1990-2$ & Suez Canal area & CS & $\begin{array}{l}\text { Healthy blood volunteers resident to } \\
\text { rural area with high schistosomiasis }\end{array}$ & 271 & $14.4 \%$ & N/A \\
\hline El Gohary,95 [36] & $1990-2$ & North Sinai, frontier & CS & Bedouin population with low schistosomiasis & 148 & $15.5 \%$ & N/A \\
\hline Darwish,95 [55] & N/A & N/A & CS & $\begin{array}{l}\text { Healthy villagers and non-professional blood } \\
\text { donors }\end{array}$ & 188 & $21.8 \%$ & $\mathrm{~N} / \mathrm{A}$ \\
\hline Darwish,96 [56] & 1994 & Kalama, Qaluobyia, Lower Egypt & CS & Village residents: $1-3$ years & 12 & $0.0 \%$ & N/A \\
\hline Darwish,96 [56] & 1994 & Kalama, Qaluobyia, Lower Egypt & CS & Village residents: 4-9 years & 21 & $0.0 \%$ & N/A \\
\hline Darwish,96 [56] & 1994 & Kalama, Qaluobyia, Lower Egypt & CS & Village residents: $10-19$ years & 46 & $8.0 \%$ & N/A \\
\hline Darwish,96 [56] & 1994 & Kalama, Qaluobyia, Lower Egypt & CS & Village residents: 20-39 years & 29 & $20.0 \%$ & N/A \\
\hline Darwish,96 [56] & 1994 & Kalama, Qaluobyia, Lower Egypt & CS & Village residents: $40-67$ years old & 47 & $51.0 \%$ & N/A \\
\hline El-Sayed,97 [57] & $1993-4$ & Sinai, frontier & CS & $\begin{array}{l}\text { Immigrants to a newly reclaimed area endemic } \\
\text { for schistosomiasis }\end{array}$ & 506 & $10.3 \%$ & $\mathrm{~N} / \mathrm{A}$ \\
\hline
\end{tabular}


Table 2 Studies reporting hepatitis C virus prevalence among the general population in Egypt (Continued)

\begin{tabular}{|c|c|c|c|c|c|c|c|}
\hline Nafeh,00 [58] & N/A & Assuit, Upper Egypt & CS & Village residents $\geq 5$ years of age (overall) & 6,031 & $8.7 \%$ & $5.4 \%$ \\
\hline Nafeh,00 [58] & N/A & Assuit, Upper Egypt & CS & Village residents $\leq 30$ years & 4,164 & $3.6 \%$ & $2.0 \%$ \\
\hline Nafeh,00 [58] & N/A & Assuit, Upper Egypt & CS & Village residents $>30$ years & 1,867 & $20.0 \%$ & $12.9 \%$ \\
\hline Abdel-Aziz,00 [59] & 1997 & Aghour El Soughra, Qaluobyia, Lower Egypt & CS & Village residents $\geq 5$ years old (overall) & 3,999 & $24.3 \%$ & $14.8 \%$ \\
\hline Abdel-Aziz,00 [59] & 1997 & Aghour El Soughra, Qaluobyia, Lower Egypt & CS & Village residents: $\leq 20$ years & 2,105 & $9.3 \%$ & N/A \\
\hline Abdel-Aziz,00 [59] & 1997 & Aghour El Soughra, Qaluobyia, Lower Egypt & CS & Village residents $>20$ years & 1,894 & $41.0 \%$ & N/A \\
\hline Darwish,01 [60] & $1994-5$ & Kalama, Qaluobyia, Lower Egypt & CS & Village residents $>10$ years of age & 796 & $40.0 \%$ & N/A \\
\hline El-Sadawy,04 [61] & N/A & Sharkia, Lower Egypt & CS & & 842 & $27.4 \%$ & $7.4 \%$ \\
\hline Arafa,05 [62] & $2002-3$ & Zawiat Razin, Menoufia, Lower Egypt & CS & & 4,020 & $11.8 \%$ & N/A \\
\hline Arafa,05 [62] & $2002-3$ & Zawiat Razin, Menoufia, Lower Egypt & CS & Village residents: under 20 years old & 1,759 & $2.8 \%$ & N/A \\
\hline Arafa,05 [62] & $2002-3$ & Zawiat Razin, Menoufia, Lower Egypt & CS & Village residents: 20 and over years old & 2,252 & $18.9 \%$ & N/A \\
\hline Mohamed,06 [63] & 2002 & Zawiat Razin, Menoufia, Lower Egypt & CS & Village residents $18-65$ years of age & 2,425 & $18.5 \%$ & N/A \\
\hline Eassa,07 [64] & $2006-7$ & Zagazig district, Sharkia, Lower Egypt & CS & Village households & 304 & $10.9 \%$ & N/A \\
\hline Aguilar,08 [65] & N/A & Fakkous and 8 surrounding villages & CS & Village residents: males & 78 & $51.3 \%$ & $38.5 \%$ \\
\hline Aguilar,08 [65] & N/A & $\begin{array}{l}\text { Fakkous and } 8 \text { surrounding villages, } \\
\text { Sharkia, Lower Egypt }\end{array}$ & CS & Village residents: females & 81 & $42.0 \%$ & $29.6 \%$ \\
\hline \multicolumn{8}{|l|}{ Children } \\
\hline Abdel-Wahab,94 [53] & 1992 & Menoufia, Lower Egypt & CS & Rural male primary school children & 190 & $12.1 \%$ & N/A \\
\hline El-Sherbini,03 [66] & 1994 & N/A & CS & Village school children 6-15 years old & 294 & $5.8 \%$ & $2.4 \%$ \\
\hline Mohamed,06 [67] & 1997 & Nile River Delta, Lower Egypt & CS & Village children $5-18$ years old & 1,823 & $8.2 \%$ & N/A \\
\hline Mohamed,06 [67] & 1997 & Assuit, Upper Egypt & CS & Village children $5-18$ years old & 2,808 & $2.5 \%$ & N/A \\
\hline El Sherbini,07 [68] & 2002 & Tanta, Gharbia, Lower Egypt & CS & School children & 470 & $2.1 \%$ & $0.8 \%$ \\
\hline Barakat,11 [69] & 2005 & Alexandria city, Alexandria & PBS & School children & 500 & $5.8 \%$ & $4.4 \%$ \\
\hline \multicolumn{8}{|l|}{ Healthy individuals } \\
\hline El-Sayed,96 [70] & 1994 & South Sinai, frontier & CS & Tourism workers & 740 & $14.3 \%$ & N/A \\
\hline Mohamed,96 [71] & N/A & N/A & CS & Egyptians applying for work abroad & 5,071 & $31.5 \%$ & N/A \\
\hline Mohamed,96 [71] & N/A & N/A & CS & Egyptians applying for work abroad: Females & N/A & $13.2 \%$ & N/A \\
\hline Mohamed,96 [71] & N/A & N/A & CS & Egyptians applying for work abroad: Males & N/A & $34.0 \%$ & N/A \\
\hline Gohar,95 [72] & N/A & N/A & CS & & 15 & $13.3 \%$ & N/A \\
\hline Halim,99 [73] & 1996 & Cairo city, Cairo & CS & Healthy staff of the university & 50 & $6.0 \%$ & N/A \\
\hline Hassan,01 [74] & $1995-6$ & Cairo city, Cairo & CS & Healthy individuals visiting hospitalized friends & 35 & $42.9 \%$ & N/A \\
\hline Strickland,02 [75] & N/A & Nile River Delta, Lower Egypt & CS & & 212 & $46.7 \%$ & $36.3 \%$ \\
\hline El-sayed,06 [76] & 2002 & Cairo city, Cairo & CS & & 36 & $8.3 \%$ & $2.8 \%$ \\
\hline El Bassuoni,08 [77] & N/A & N/A & CS & & 10 & $30.0 \%$ & N/A \\
\hline
\end{tabular}


Table 2 Studies reporting hepatitis C virus prevalence among the general population in Egypt (Continued)

\begin{tabular}{|c|c|c|c|c|c|c|c|}
\hline Salama,09 [78] & N/A & N/A & CS & & 20 & $5.0 \%$ & N/A \\
\hline \multicolumn{8}{|c|}{ Army recruits/Fire brigade personnel } \\
\hline Farghaly,93 [79] & N/A & N/A & CS & Army recruits & 726 & $33.0 \%$ & N/A \\
\hline Abdel-Wahab,94 [53] & 1992 & Lower Egypt & CS & Army recruits & 300 & $22.1 \%$ & N/A \\
\hline Quinti,95 [34] & $1992-4$ & Alexandria city, Alexandria & CS & Fire brigade personnel & 541 & $39.0 \%$ & N/A \\
\hline \multicolumn{8}{|c|}{ Other general populations } \\
\hline El-Ahmady,94 [10] & N/A & Cairo city, Cairo & CS & & 292 & $24.3 \%$ & N/A \\
\hline El-Sadawy,04 [61] & N/A & Sharkia, Lower Egypt & PBS & & 1,422 & $25.8 \%$ & $7.7 \%$ \\
\hline El-Sadawy,04 [61] & N/A & Sharkia, Lower Egypt & PBS & General population in urban areas & 580 & $23.4 \%$ & N/A \\
\hline El-Sadawy,04 [61] & N/A & Sharkia, Lower Egypt & PBS & General population: $<20$ years & 414 & $4.8 \%$ & N/A \\
\hline El-Sadawy,04 [61] & N/A & Sharkia, Lower Egypt & PBS & General population: 20 - 30 years & 163 & $14.1 \%$ & N/A \\
\hline El-Sadawy,04 [61] & N/A & Sharkia, Lower Egypt & PBS & General population: $30-40$ years & 253 & $30.0 \%$ & N/A \\
\hline El-Sadawy,04 [61] & N/A & Sharkia, Lower Egypt & PBS & General population: $>40$ years & 592 & $41.9 \%$ & N/A \\
\hline Mohamed,04 [80] & 1996-7 & 10 governorates & PBS & General population & 7,357 & $13.5 \%$ & N/A \\
\hline El Zanaty,09 [1] & 2008 & Nationwide & PBS & & 11,126 & $14.7 \%$ & $9.8 \%$ \\
\hline
\end{tabular}

CS convenience sampling, PBS probability-based sampling, N/A not available. 


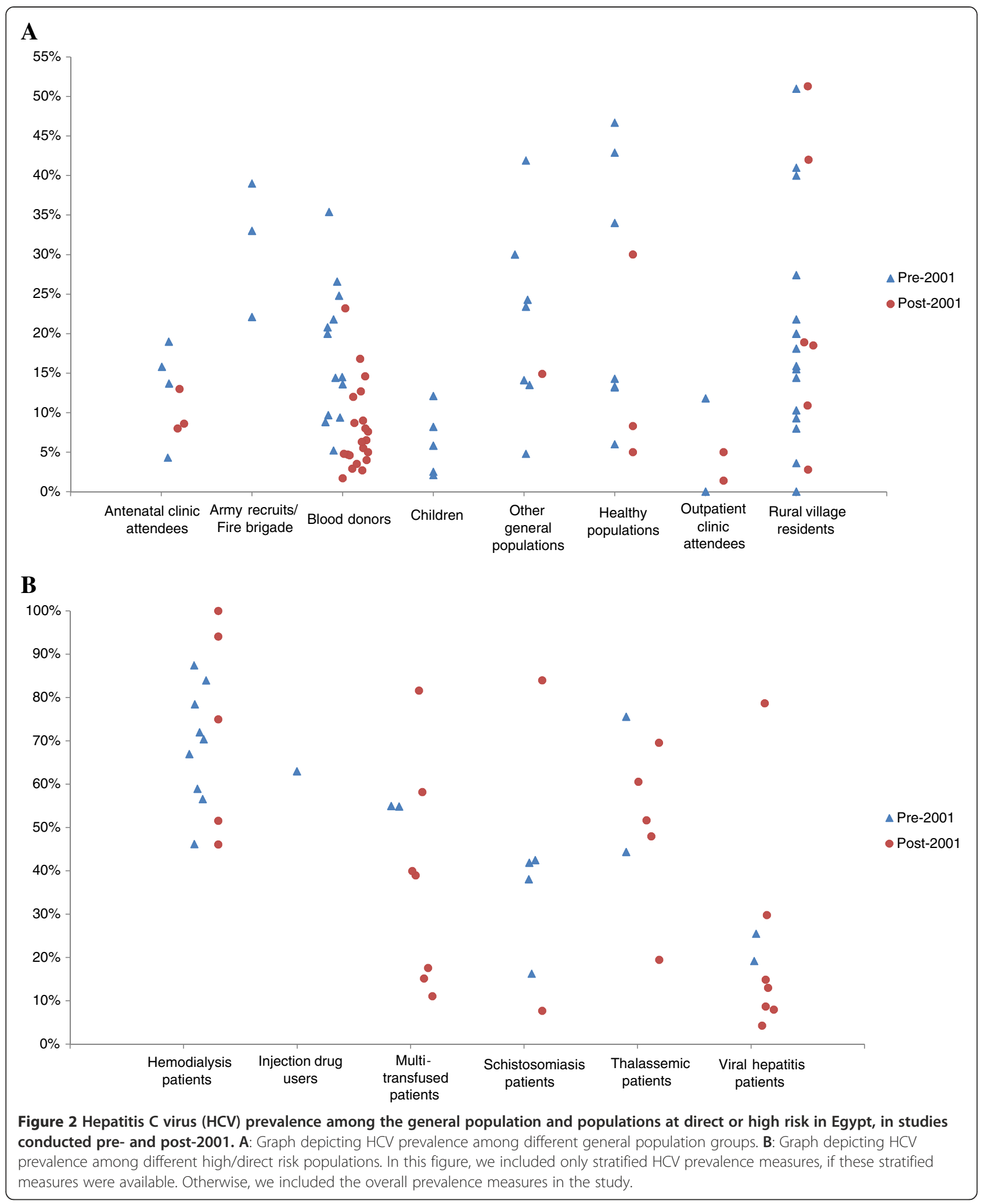


Table 3 Studies reporting prevalence of hepatitis C virus among populations at direct or high risk of exposure in Egypt

\begin{tabular}{lllll}
\hline Citation & Year Location & Sampling & $\begin{array}{c}\text { Population characteristic } \\
\text { size }\end{array}$ & $\begin{array}{c}\text { Sample } \\
\text { sizo-prevalence }\end{array}$ \\
prevale
\end{tabular}

\section{Viral hepatitis patients}

El-Ahmady,94 [10]

N/A Cairo city, Cairo

CS

El-Gohary,94 [83]

N/A Suez city, Suez

CS

El Gaafary,05 [84]

2002 Cairo city, Cairo

CS

Meky,06 [85]

Zakaria,07 [86]

2002-5 Nile River Delta, Lower Egypt

CS

Kalil,10 [87]

2001-2 Giza, Upper Egypt

Talaat, $10[88]$

2004-5 Assuit, Upper Egypt

2001-4 Alexandria city, Alexandria/ Abassia, Cairo/Mahalla, Gharbia, Lower Egypt/Qena and Aswan, Upper Egypt

Eldin,10 [89]

$$
\text { N/A N/A }
$$

Badawy,12 [90]

N/A Cairo city, Cairo

\section{Multi-transfused patients}

\begin{tabular}{|c|c|c|}
\hline Khalifa,93 [18] & 1990-1 & Cairo city, Cairo \\
\hline Abdel-Wahab,94 [53] & 1992 & Cairo city, Cairo \\
\hline Said,09 [91] & $\mathrm{N} / \mathrm{A}$ & Cairo city, Cairo \\
\hline Said,09 [91] & N/A & Cairo city, Cairo \\
\hline Salama,09 [78] & N/A & Cairo city, Cairo \\
\hline Kalil,10 [87] & $2004-5$ & Assuit, Upper Egypt \\
\hline Tonbary,10 [92] & $2000-8$ & $\begin{array}{l}\text { Mansoura, Dakahlia, } \\
\text { Lower Egypt }\end{array}$ \\
\hline El-Faramawy,12 [93] & N/A & Qena, Upper Egypt \\
\hline Abdelwahab,12 [94] & N/A & Cairo city, Cairo \\
\hline \multicolumn{3}{|l|}{ Thalassemic patients } \\
\hline El-Nanawy,95 [19] & N/A & Alexandria city, Alexandria \\
\hline El Gohary,95 [36] & $1990-2$ & Cairo city, Cairo \\
\hline Khalifa,04 [95] & $2000-3$ & Cairo city, Cairo \\
\hline Abdalla,06 [96] & 2005 & $\begin{array}{l}\text { Cairo city, Cairo / Banha, } \\
\text { Qalubiya, Lower Egypt }\end{array}$ \\
\hline Omar,11 [97] & N/A & Cairo city, Cairo \\
\hline Mansour,12 [98] & 2009-10 & $\begin{array}{l}\text { Mansoura, Dakahlia, } \\
\text { Lower Egypt }\end{array}$ \\
\hline El-Faramawy,12 [93] & /A & Qena, Upper Egypt \\
\hline
\end{tabular}

\section{Schistosomiasis patients}

$\begin{array}{lrll}\text { Bassily,92 [99] } & \text { N/A } & \text { Nile River Delta, Lower Egypt } \\ \text { El-Nanawy,95 [19] } & \text { N/A } & \text { Alexandria city, Alexandria } \\ \text { El-Zayadi,97 [39] } & \text { N/A } & \text { N/A } \\ \text { Zekri,02 [100] } & 1998-00 & \text { Cairo city, Cairo } \\ \text { Arafa,05 [62] } & 2002-3 & \begin{array}{l}\text { Zawiat Razin, Menoufia, } \\ \text { Lower Egypt }\end{array} \\ \text { El-Sabah,11 [101] } & \text { N/A } & \begin{array}{l}\text { Rural area, Cairo/ Gharbia, } \\ \text { Lower Egypt }\end{array} \\ \text { El-Sabah,11 [101] } & \text { N/A } & \begin{array}{l}\text { Rural area, Cairo/ Gharbia, } \\ \text { Lower Egypt }\end{array}\end{array}$

CS

CS

CS

CS

CS

CS

CS

CS

CS

CS

CS

CS

CS

CS

CS

CS

$\begin{array}{crrr} & 51 & 25.5 \% & \mathrm{~N} / \mathrm{A} \\ & 140 & 19.2 \% & \mathrm{~N} / \mathrm{A} \\ & 309 & 14.9 \% & \mathrm{~N} / \mathrm{A} \\ & 47 & 78.7 \% & 70.2 \% \\ \text { Children with viral hepatitis } & 200 & 13.0 \% & \mathrm{~N} / \mathrm{A} \\ & 150 & 8.7 \% & 8.7 \% \\ & 4,189 & 29.8 \% & \mathrm{~N} / \mathrm{A}\end{array}$

Male military recruits with viral hepatitis

Multi-transfused children

Multi-transfused children

Multi-transfused children with hematological disorders

Multi-transfused children with hematological malignancies

Multi-transfused children

Multi-transfused children

Multi-transfused children

Multi-transfused children

Multi-transfused children

Children with thalassemia

Children with thalassemia

Children with thalassemia

Children with thalassemia

Children with thalassemia

$19.5 \%$ 
Table 3 Studies reporting prevalence of hepatitis C virus among populations at direct or high risk of exposure in Egypt (Continued)

\begin{tabular}{|c|c|c|c|c|c|c|c|}
\hline & & & & $\begin{array}{l}\text { Schistosomiasis patients } \\
\text { treated orally up to } \\
8 \text { years ago }\end{array}$ & & & \\
\hline \multicolumn{8}{|c|}{ Hemodialysis patients } \\
\hline Hassan,93 [102] & N/A & N/A & CS & & 105 & $67.0 \%$ & N/A \\
\hline Abdel-Wahab,94 [53] & 1992 & Cairo city, Cairo & CS & & 78 & $46.2 \%$ & $\mathrm{~N} / \mathrm{A}$ \\
\hline El-Ahmady,94 [10] & N/A & Cairo city, Cairo & CS & & 25 & $84.0 \%$ & N/A \\
\hline El Gohary,95 [36] & $1990-2$ & $\begin{array}{l}\text { Suez city, Suez and } \\
\text { Ismailia, Lower Egypt }\end{array}$ & CS & & 108 & $70.4 \%$ & N/A \\
\hline Gohar,95 [72] & N/A & N/A & CS & & 64 & $87.5 \%$ & $N / A$ \\
\hline Hassan,00 [103] & 1996 & Cairo city, Cairo & CS & & 210 & $59.0 \%$ & N/A \\
\hline Shatat,00 [104] & 1999 & N/A & CS & & 83 & $78.5 \%$ & N/A \\
\hline Gad,02 [105] & 1998 & Ismailia, Lower Egypt & CS & & 47 & $72.0 \%$ & N/A \\
\hline Zekri,02 [100] & 1998-00 & Cairo city, Cairo & CS & & 30 & $56.6 \%$ & N/A \\
\hline El Yazeed,06 [106] & $2002-4$ & Cairo city, Cairo & CS & & 40 & $100.0 \%$ & N/A \\
\hline Kandil,07 [22] & $2004-6$ & Cairo city, Cairo & CS & & 31 & $51.6 \%$ & N/A \\
\hline Hammad,09 [25] & 2008 & $\begin{array}{l}\text { Mansoura, Dakahlia, } \\
\text { Lower Egypt }\end{array}$ & CS & & 34 & $94.1 \%$ & N/A \\
\hline Attia,10 [107] & $2008-9$ & Cairo city, Cairo & CS & & 206 & $46.1 \%$ & N/A \\
\hline Ibrahim,10 [108] & 2007 & Cairo city, Cairo & CS & & 100 & $75.0 \%$ & N/A \\
\hline \multicolumn{8}{|c|}{ Injection Drug Users (IDUs) } \\
\hline El-Ghazzawi,95 [109] & N/A & Alexandria city, Alexandria & CS & & 100 & $63.0 \%$ & N/A \\
\hline
\end{tabular}

CS convenience sampling, N/A not available, PAT parenteral antischistosomiasis therapy.

Overall, the average HCV prevalence among schistosomiasis patients was about $38 \%$. A recent study by Sabah et al. reported a prevalence of $84.0 \%$ among schistosomiasis patients treated with PAT 20 to 30 years ago, and a prevalence of $7.7 \%$ among schistosomiasis patients treated orally up to 8 years ago [101].

We were able to identify only one study conducted among IDUs in Egypt [109]. The study was conducted in Alexandria, among 100 IDUs, and HCV prevalence was reported to be $63.0 \%$ [109].

Figure 2B depicts the range of prevalence within each subgroup in studies conducted pre and post-2001. No distinct pattern can be discerned in the distribution of $\mathrm{HCV}$ prevalence pre- and post-2001 within each of the different subgroups.

\section{Prevalence of $\mathrm{HCV}$ among populations at indirect or intermediate risk of exposure}

Populations at indirect or intermediate risk of exposure to $\mathrm{HCV}$ were classified into the following subcategories: diabetic patients, hospital outpatient attendees, hospitalized populations, household contacts of index cases (HCV positive cases), sexually transmitted infection (STI) patients, periodontal disease patients, prisoners, and populations working in select professions. Details are shown in Table S2, see Additional file 1.
Considerable $\mathrm{HCV}$ prevalence was reported among diabetic children in Egypt compared to other countries. A recent study conducted in 2010, among 692 diabetic children with an average age of 10.4 years, reported a prevalence of $2.5 \%$ [110]. Previous studies conducted among diabetic children reported much higher levels of $29.4 \%$ [19] and 44.1\% [22]. In contrast, HCV prevalence among adult diabetic patients was $20.0 \%$ [100] and $60.3 \%$ [111]. Substantial HCV prevalence was also observed among patients attending hospitals, ranging between $0 \%$ [87] and 72.8\% [112]. We identified one study conducted among periodontal disease patients reporting a prevalence of 13.0\% [113]. Multiple studies were conducted investigating $\mathrm{HCV}$ prevalence among the children, spouses, and family contacts of $\mathrm{HCV}$ positive cases. Studies conducted among children of index cases usually focused on children of infected mothers to examine the vertical transmission of HCV. HCV RNA prevalence among infants born to $\mathrm{HCV}$ positive mothers ranged between $3.8 \%$ [28] and $25.0 \%$ [29]. HCV prevalence among spouses of index patients was as high as $35.5 \%$ [114]. In a study of family contacts of index patients, the prevalence was 5.7\% [115].

Six studies were conducted among populations in select HCV-relevant professions (Table S2 of Additional file 1). $\mathrm{HCV}$ prevalence among health care workers averaged about $17 \%$, whereas among barbers it was $12.3 \%$ [116]. 
We were able to identify only one study among prisoners which reported a prevalence of 31.4\% [34].

The range of $\mathrm{HCV}$ prevalence within each subgroup in studies conducted pre- and post-2001 can be observed in Figure 3A. No distinct pattern can be discerned in the distribution of HCV prevalence pre- and post-2001 within each of the different subgroups.

\section{Prevalence of HCV among special clinical populations}

A large fraction of studies were conducted among different clinical populations (Table S3 of Additional file 1). Overall, $\mathrm{HCV}$ prevalence was very high across all special clinical population groups. The average $\mathrm{HCV}$ prevalence among non-Hodgkin's lymphoma (NHL) patients was roughly $41 \%$, while among orthopedic patients it was about $39 \%$. $\mathrm{HCV}$ prevalence among hepatocellular carcinoma (HCC) cases ranged between $61.0 \%$ and $90.3 \%$, with a higher prevalence observed among rural versus urban populations [117]. Three studies were conducted among pediatric cancer patients $[81,118,119]$. HCV prevalence among children with leukemia was $19.0 \%$ [118]. HCV prevalence among patients with pediatric malignancies who had just ended chemotherapy was 39.6\% [119]. More recently, Sharaf-Eldeen et al. reported HCV prevalence of $43.0 \%$ among children with malignant cancers [81].

No distinct pattern can be discerned in the distribution of HCV prevalence pre- and post-2001 within each of the different special clinical population subgroups (Figure 3B).

\section{RNA prevalence}

Measures of RNA prevalence are included in Tables 2-3, and S2-S3 of Additional file 1. RNA prevalence was high across studies in the different population groups. Higher RNA prevalence was observed among studies conducted among high risk groups and special clinical populations compared to the general population and indirect or intermediate risk groups. Overall, the average RNA prevalence among those $\mathrm{HCV}$-antibody positive was approximately $60 \%$.

\section{Risk factors of HCV}

Increasing age, a history of PAT, and residing in rural areas were by far the most common risk factors or associations with $\mathrm{HCV}$ infection across studies [26,62]. Other common risk factors were related to healthcare settings such as history of blood transfusions, invasive procedures, injections, perinatal care, and dental work $[15,61,62,69,71,82,87]$. Saleh et al. reported a greater risk for incident infection among women whose babies were delivered by a physician rather than by a nurse or a traditional birth attendant, in a health facility rather than at their home, and in women having complicated vaginal deliveries [15]. Among children, incident infection was associated with hospitalization and low birth weight
[17]. Community and informal health provider related exposures such as circumcision, cautery, and injections were also associated with HCV infection [62,82,120]. A number of studies have also suggested intrafamilial transmission though the exact exposures responsible are not clear $[14,121]$.

\section{Time trend analysis General population model}

Twenty six of the 87 general population studies had the year of data collection missing. The results of our paired t-test identified a mean difference of 3.1 years $(95 \% \mathrm{CI}$ : 2.6 to 3.6) between the year of publication and the year of data collection, for studies with both values present. We applied this time lag to estimate the year of data collection for studies missing this value.

In the univariate linear regression analyses for each subgroup separately, blood donors were the only subgroup showing a statistically significant change in $\mathrm{HCV}$ prevalence over time ( $\mathrm{p}$-value of 0.001). Table S4, see Additional file 1, reports the results of the univariate analyses.

In the multivariate linear regression analysis for the combined general population subgroups, there was no evidence of a statistically significant decline in $\mathrm{HCV}$ prevalence over time (p-value of 0.215). HCV prevalence in the general population changed at a rate of $-0.24 \%$ per year (95\% CI: -0.63 to 0.14 ). Figure 4 displays the trend in $\mathrm{HCV}$ prevalence with time in each of the eight general population subgroups.

\section{Direct or high risk population model}

Twenty five of the 46 direct or high risk population studies had the year of data collection missing. The results of the paired $t$-test identified a mean difference of 3.3 years (95\% CI: 4.0 to 2.6) between the year of publication and the year of data collection. We applied this time lag to estimate the year of data collection for studies missing this value.

As was the case for the general population univariate and multivariate analyses, there was no evidence of a statistically significant decline in HCV prevalence over time for each high risk population subgroup (results not shown) and in the high risk population as a whole (p-value of 0.426). HCV prevalence in the high risk population declined at a rate of $-0.38 \%$ per year $(95 \%$ CI: -1.35 to 0.58 ). Figure S5, of Additional file 1, displays the trend in $\mathrm{HCV}$ prevalence with time in each of the five high risk subgroups. IDUs were excluded from this figure as there was only one observation.

\section{Discussion}

We have systematically reviewed $\mathrm{HCV}$ infection prevalence and incidence across the different population groups in 


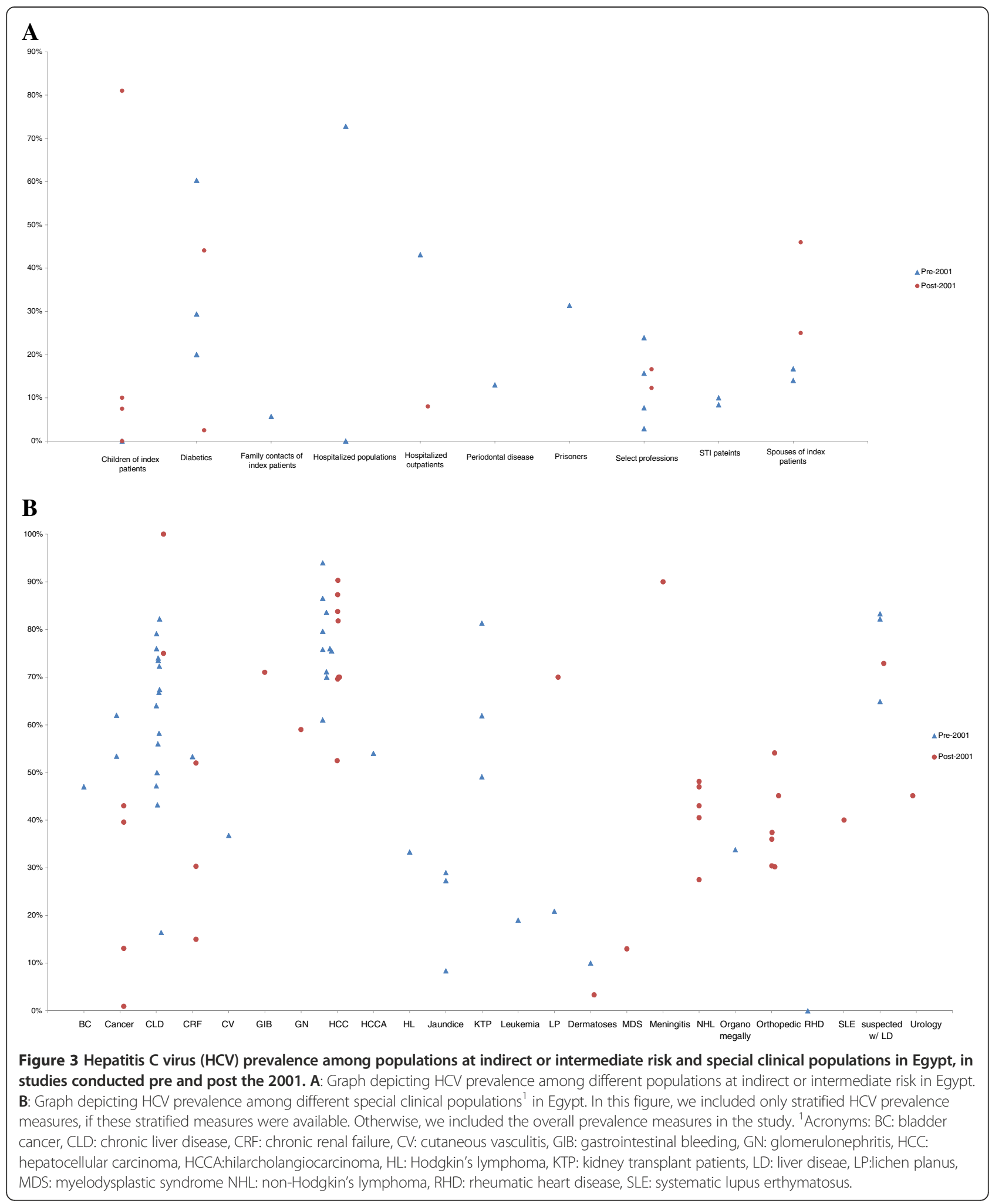

Egypt. The results of our study indicate that Egypt is enduring a large HCV disease burden, and is likely to be the most affected nation worldwide by this infection. HCV prevalence and incidence across the diverse population groups were found to be much higher than those in other countries around the globe $[3,122,123]$. This makes HCV and its complications one of the leading public health problems that Egypt has to confront today. 


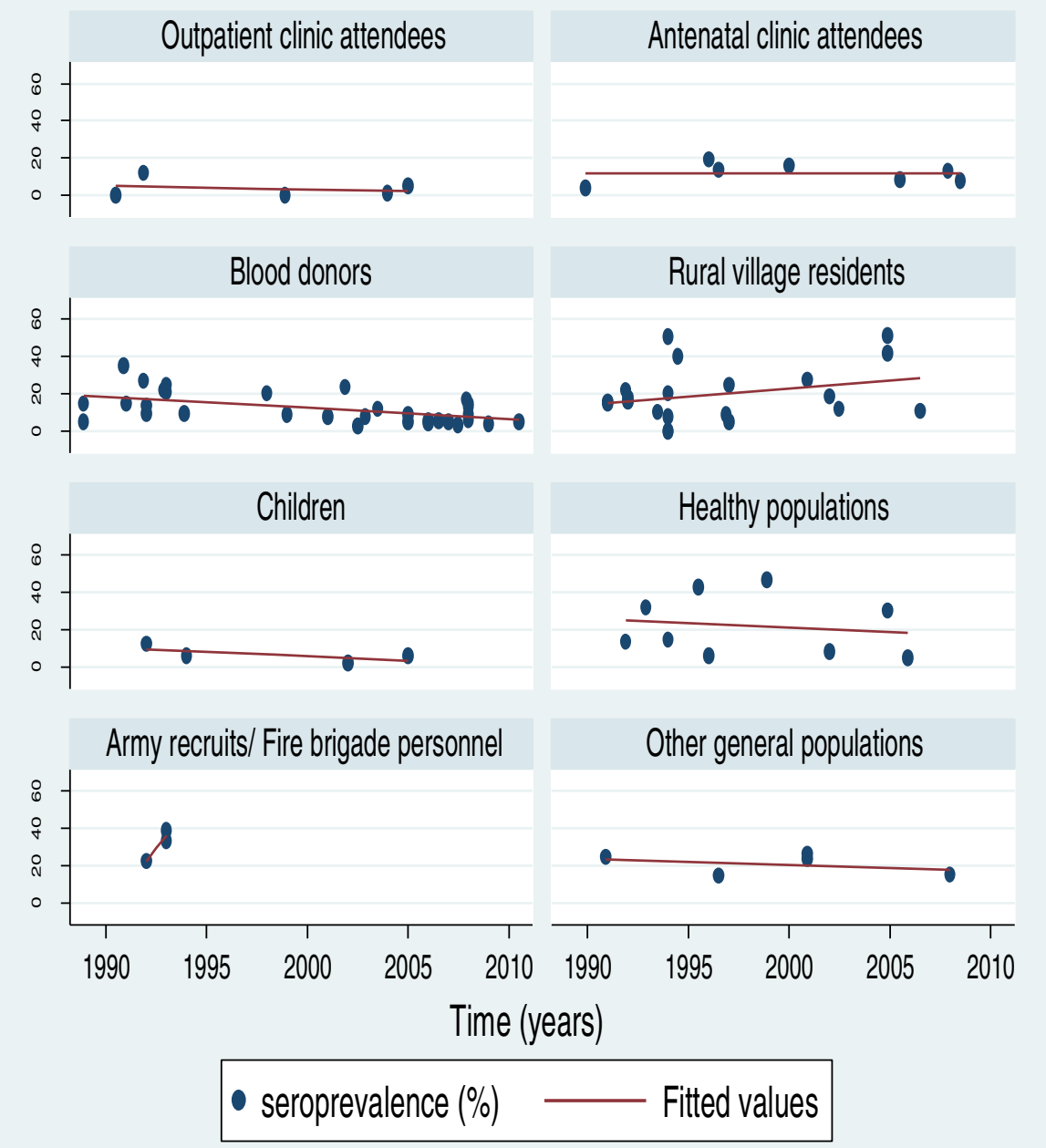

Figure 4 Time trend of hepatitis C prevalence among the different general population subgroups in Egypt.

The results of this synthesis indicate that Egypt has endured a large HCV epidemic at the national level, and that there is substantial endemic HCV transmission in this country. HCV prevalence is at high levels across essentially all population groups, demonstrating the expansive nature of this epidemic, and that it is not isolated to specific population groups or geographic areas.

Despite being a major driver of $\mathrm{HCV}$ incidence and prevalence in many countries [124], the relative contribution of IDUs to HCV incidence and prevalence in Egypt is much smaller than that in other countries. This is because of the specific context of HCV in Egypt, where $\mathrm{HCV}$ transmission is associated with medical exposures in the context of a general population $\mathrm{HCV}$ epidemic. Still, there is a contribution from injecting drug use to $\mathrm{HCV}$ transmission that is, in terms of absolute scale, comparable to other countries. The prevalence of injecting drug use in Egypt is estimated to be 0.21\% [125], and according to the only study we found, HCV prevalence among IDUs is 63\% [109]. Considering that HCV prevalence in the population is $14.7 \%$ [1], injecting drug use may explain at most only about $1 \%$ of the national HCV prevalence in Egypt.

It is widely believed that the PAT campaigns to control schistosomiasis are the major drivers of the HCV epidemic in Egypt [5]. During the early twentieth century, schistosomiasis was highly prevalent in Egypt, especially in rural areas [6]. From the 1950s to the early 1980s, the Egyptian Ministry of Health led large-scale campaigns to control the disease [6]. Millions of people were treated with intravenous injections of tartar emetic, before an oral drug replaced this standard of care across the country in the 1980s [5]. Reuse of glass syringes and lax sterilization practices during PAT campaigns appear to have caused widespread infection with $\mathrm{HCV}$, which by the 1990s, had replaced schistosomiasis as the primary cause of liver disease in Egypt $[6,126]$. 
Our study supports a major role for the PAT campaigns in driving HCV incidence. Different studies have shown a dramatic increase in $\mathrm{HCV}$ prevalence with age; a cohort effect that may be explained, at least in part, by the early association between PAT and HCV transmission [26,60-62,82,127]. Our results also highlight gender and geographical differences in $\mathrm{HCV}$ prevalence $[36,64,71,75,128,129]$, with higher prevalence observed in males and rural dwellers compared to females and individuals living in urban areas. These differences may also be in part attributed to the PAT campaigns, as rural areas and males were more affected by the schistosomiasis disease burden and hence were main targets of these campaigns [5].

However, the totality of the evidence synthesized here suggests that the PAT campaigns are one driver among others of HCV transmission in Egypt, and that substantial $\mathrm{HCV}$ transmission is still ongoing. High HCV prevalence is found among hospitalized and the special clinical populations; populations that have experienced various facilitybased medical procedures. Elevated $\mathrm{HCV}$ prevalence is also found among individuals exposed to even minor medical care procedures, within and beyond established health care facilities. Community studies have found strong correlations between $\mathrm{HCV}$ infection and different medical exposures such as injections, blood transfusions, surgical procedures, perinatal care, and dental treatment $[15,61,62,69,71,82,87]$.

HCV prevalence among children, in particular, highlights the ongoing transmission of $\mathrm{HCV}$ in Egypt. Not only were these children born well after the end of the PAT campaigns, but also a large fraction of them were born after the implementation of the more stringent infection control measures in the country. Nevertheless, considerable prevalence levels are found among children in multiple studies. These studies suggest that children have been exposed to HCV vertically through mother-to-child transmission [24,25,27-29] (high RNA prevalence was documented among infants of $\mathrm{HCV}$ positive mothers, ranging between $3.8 \%$ and $11.1 \%$ [24,25,27-29,130,131]), or horizontally possibly through household exposures $[14,114,115,121,131]$. Medical exposures to HCV at a very young age have been also indicated [19,22,110,132]. High HCV levels were reported among thalassemic children $[19,96,133]$, children on hemodialysis $[22,132]$ and diabetic children [19,22,110].

$\mathrm{HCV}$ prevalence among the mothers of infected children, who tend to be young themselves, has been also associated with medical exposures and/or household exposures [24,25,27,28]. Injecting drug use is unlikely to contribute much to $\mathrm{HCV}$ prevalence among these mothers, given the context of the $\mathrm{HCV}$ epidemic in Egypt. Moreover, injecting drug use among women in the Middle East and North Africa region is believed to be marginal with only about $10 \%$ or less of IDUs being females in this region [134-136].

Results of our time trend analysis suggest that, contrary to expectations, there appears to be a small decline, though statistically not significant, in $\mathrm{HCV}$ prevalence over time in the general population and high risk population in Egypt. In the univariate subgroup-specific analyses, only blood donors have shown a statistically significant decline in HCV prevalence. However, this decline is difficult to interpret since recruitment of blood donors changed over time, particularly by excluding HCV positive individuals. While it can take a long time for the prevalence of HCV to decline after the PAT exposures, this fact may not be sufficient to explain the lack of tangible decline. Egypt's population has almost doubled in the past two to three decades since the epidemic was first discovered, and well after the end of the PAT campaigns. The large influx of uninfected birth cohorts does not appear to have reduced $\mathrm{HCV}$ prevalence, possibly suggesting that $\mathrm{HCV}$ incidence has not declined as expected following the end of the PAT campaigns and adoption of more stringent infection control measures.

Another potential explanation for the lack of substantial decline in incidence is the very high baseline HCV prevalence in the country. For a given mode of transmission, such as reuse of unclean needles or syringes, the transmission risk is dependent on the probability that the needle/ syringe was used previously on an $\mathrm{HCV}$ infected person, which is HCV RNA prevalence. Even if the prevalence of the modes of transmissions in Egypt today is similar to other neighboring countries, the high background prevalence can drive much more incidence. In neighboring Libya for example, with an HCV RNA prevalence of less than $1 \%[134,137]$, a reuse of an unclean needle is more than ten-fold less likely to lead to an HCV transmission than in Egypt where HCV RNA prevalence is 9.8\% [1].

Our study identified the lack of an empirical nationallyrepresentative incidence study. It is a priority to document current $\mathrm{HCV}$ incidence rate in the population, which continues to be the most critical open question in $\mathrm{HCV}$ epidemiology in Egypt today, and is subject to much debate $[127,138]$. A recent mathematical modeling study, based on the EDHS data, estimated that the average HCV incidence rate over the lifetime of the living Egyptian population cohort to be 6.9 per 1,000 person-year [7]. This estimate however does not capture the temporal trend in $\mathrm{HCV}$ incidence rate and may not be representative of the current level of $\mathrm{HCV}$ transmission. A recent study suggested that current $\mathrm{HCV}$ incidence rate is about 2.0 per 1000 person-year [127].

While the evidence for an epidemic at the national level is overwhelming, some of the potential drivers of this epidemic, such as the PAT campaigns and contaminated blood, are no longer present. Therefore, it seems plausible 
that $\mathrm{HCV}$ incidence rate has declined drastically in the last two decades since the discovery of the epidemic in 1991-1992 [30,139], as Breban et al. have recently suggested through their incidence estimate [127]. Nevertheless, our study could not identify a signature for a major reduction in incidence, and the totality of evidence points towards substantial ongoing $\mathrm{HCV}$ transmission, though the precise scale of which is not yet known.

In terms of limitations, there was an element of subjectivity in classifying different studies into different population subgroups. For example, studies conducted in rural children were classified under children even though they fall into two subgroups: rural populations and children. Furthermore, there was variability in the diagnostic assays used across the studies. Earlier studies typically reported the use of 1st and 2nd generation ELISA tests, which lack the sensitivity and specificity of the 3rd generation ELISA tests. Such variability in assays may impact the representativeness of the reported prevalence measures. There was also considerable variability in methodology and quality among the studies assessed. Most of the studies identified were cross-sectional or case-control in study design. Sample size varied widely across studies. The sampling was most often convenience sampling, though several studies have used probability-based samples $[1,61,69]$, most notably the EDHS conducted in 2008 [1]. For a fraction of the studies the year of data collection was not available but was estimated using the year of publication, this may affect the time trend analysis. Given that only statistically significant risk factors were extracted, this may have introduced a positive-association selection bias in our reporting of risk factors.

\section{Conclusions}

Our study highlights that Egypt is confronted with an $\mathrm{HCV}$ disease burden of historical proportions. An $\mathrm{HCV}$ epidemic at the national level must have occurred with substantial transmission still ongoing today. As opposed to other countries where HCV dynamics is focused in specific high risk groups, such as IDUs, HCV transmission in Egypt has reached diverse population groups including those not conventionally identified to be at risk of infection. HCV transmission appears to be focused in formal and informal health care settings, though transmission may be occurring in the community and at the household level, but through poorly-identified exposures.

$\mathrm{HCV}$ prevention in Egypt must be a national priority. Policymakers and public health and medical care stakeholders need to introduce and implement further prevention measures targeting the iatrogenic transmission routes, such as very stringent infection control practices. Scientific research needs to be expanded to measure current $\mathrm{HCV}$ incidence rate and to identify precisely the modes of $\mathrm{HCV}$ transmission in medical care, community, and household settings. Such studies will pave the way for effective prevention interventions that can be developed, experimentally tested, and implemented. It is also essential to develop cost-effective strategies for treatment and case management of the large pool of 5-7 million chronically infected persons with $\mathrm{HCV}$ in Egypt.

\section{Additional file}

Additional file 1: Table S1. PRISMA Checklist. Table S2. Prevalence of hepatitis $C$ virus among populations at indirect or intermediate risk of exposure in Egypt. Table S3. Prevalence of hepatitis $C$ virus among special clinical populations in Egypt. Table S4. Hepatitis C virus time trend analysis for each general population subgroup. Figure S5. Hepatitis $C$ virus time trend analysis among populations at direct or high risk of exposure.

\section{Abbreviations}

HCV: Hepatitis C virus; EDHS: Egyptian demographic health survey; PAT: Parenteral antischistosomiasis therapy; IDU: Injecting drugs use; STI: Sexually transmitted infection; NHL: Non-Hodgkin's lymphoma; HCC: Hepatocellular carcinoma; PBS: Probability-based sampling; CS: Convenience sampling; HCCA: Hilarcholangiocarcinoma; KTP: Kidney transplant patient; LP: Lichen planus; HL: Hodgkin's lymphoma; CV: Cutaneous vasculitis; CLD: Chronic liver disease; BC: Bladder cancer; Rheumatic HD: Rheumatic heart disease; CRF: Chronic renal failure; LD: Liver disease; GIB: Gastrointestinal bleeding; SLE: Systemic lupus erythematosus; MDS: Myelodysplastic syndrome; GN: Glomerulonephritis.

\section{Competing interests}

The authors declare that they have no competing interests.

\section{Authors' contributions}

YM and SR conducted the literature review and data retrieval. YM conducted extraction, analysis and wrote the first draft of the paper. GM participated in study design. FDM contributed to the study design and analyses. LJA conceived and led the design of the study, analyses, and drafting of the article. All authors contributed to discussion of the results and writing of the manuscript. All authors read and approved the final manuscript.

\section{Acknowledgements}

We are grateful for the Qatar National Research Fund for supporting this work (NPRP 04-924-3-251), and the support provided by the Biostatistics, Epidemiology, and Biomathematics Research Core at the Weill Cornell Medical College in Qatar. The authors are also grateful for the valuable suggestions and comments by the reviewers of this article.

\section{Funding}

The Qatar National Research Fund (NPRP 04-924-3-251) and the Biostatistics, Epidemiology, and Biomathematics Research Core at the Weill Cornell Medical College- Qatar.

\section{Author details}

${ }^{1}$ Infectious Disease Epidemiology Group, Weill Cornell Medical College Qatar, Cornell University, Qatar Foundation - Education City, Doha, Qatar. ${ }^{2}$ Department of Public Health, Weill Cornell Medical College, Cornell University, New York, New York, USA. ${ }^{3}$ Vaccine and Infectious Disease Division, Fred Hutchinson Cancer Research Center, Seattle, Washington, USA. ${ }^{4}$ Department of Tropical Medicine, Medical Microbiology and Pharmacology, John A. Burns School of Medicine, University of Hawaii, Honolulu, HI 96813, Hawaii.

Received: 14 August 2012 Accepted: 19 June 2013

Published: 24 June 2013 


\section{References}

1. El-Zanaty F, Way A: Egypt Demographic and Health Survey 2008. Egyptian: Ministry of Health. Cairo: El-Zanaty and Associates, and Macro International; 2009

2. Lavanchy D: Evolving epidemiology of hepatitis $C$ virus. Clin Microbiol Infect 2011, 17(2):107-115.

3. Alter MJ: Epidemiology of hepatitis C virus infection. World J Gastroenterol 2007, 13(17):2436-2441.

4. Shepard CW, Finelli L, Alter MJ: Global epidemiology of hepatitis C virus infection. Lancet Infect Dis 2005, 5(9):558-567.

5. Frank C, Mohamed MK, Strickland GT, Lavanchy D, Arthur RR, Magder LS, El Khoby T, Abdel-Wahab Y, Aly Ohn ES, Anwar W, et al: The role of parenteral antischistosomal therapy in the spread of hepatitis $C$ virus in Egypt. Lancet 2000, 355(9207):887-891.

6. Strickland GT: Liver disease in Egypt: hepatitis C superseded schistosomiasis as a result of iatrogenic and biological factors. Hepatology 2006, 43(5):915-922.

7. Miller FD, Abu-Raddad L: Evidence of intense ongoing endemic transmission of hepatitis C virus in Egypt. Proc Natl Acad Sci USA 2010, 107(33):14757-14762.

8. Moher D, Liberati A, Tetzlaff J, Altman DG, The PG: Preferred Reporting Items for Systematic Reviews and Meta-Analyses: The PRISMA Statement. PLoS Med 2009, 6(7):e1000097.

9. Talaat M, Kandeel A, Rasslan O, Hajjeh R, Hallaj Z, El-Sayed N, Mahoney FJ: Evolution of infection control in Egypt: Achievements and challenges. Am J Infect Control 2006, 34(4):193-200.

10. el-Ahmady $\mathrm{O}$, Halim AB, Mansour $\mathrm{O}$, Salman T: Incidence of hepatitis $C$ virus in Egyptians. J Hepatol 1994, 21(4):687.

11. Elkareh S: HCv screening of donors in Governmental Blood Transfusion Centers at Menoufia Governorate (from Jan. 2008 to Oct. 2008). Vox Sang 2009, 96:89-90.

12. Eita N: Prevalence of HCV and HBV infections among blood donors in Dakahilia, Egypt. Vox Sang 2009, 96:106-107.

13. Rushdy O, Moftah F, Zakareya S: Trasmitted transfused viral infections among blood donors during years 2006 and 2007 in suez canal area, Egypt. Vox Sang 2009, 96:86-87.

14. Mohamed MK, Abdel-Hamid M, Mikhail NN, Abdel-Aziz F, Medhat A, Magder LS, Fix AD, Strickland GT: Intrafamilial transmission of hepatitis $C$ in Egypt. Hepatology 2005, 42(3):683-687.

15. Saleh DA, Shebl F, Abdel-Hamid M, Narooz S, Mikhail N, El-Batanony M, El-Kafrawy S, El-Daly M, Sharaf S, Hashem M, et al: Incidence and risk factors for hepatitis $C$ infection in a cohort of women in rural Egypt. Trans R Soc Trop Med Hyg 2008, 102(9):921-928.

16. Mostafa A, Taylor SM, El-Daly M, El Hoseiny M, Bakr I, Arafa N, Thiers V, Rimlinger F, Abdel-Hamid M, Fontanet $A$, et al: Is the hepatitis $C$ virus epidemic over in Egypt? Incidence and risk factors of new hepatitis $C$ virus infections. Liver Int 2010, 30(4):560-566.

17. Saleh DA, Shebl FM, El-Kamary SS, Magder LS, Allam A, Abdel-Hamid M, Mikhail N, Hashem M, Sharaf S, Stoszek SK, et al: Incidence and risk factors for community-acquired hepatitis $C$ infection from birth to 5 years of age in rural Egyptian children. Trans R Soc Trop Med Hyg 2010, 104(5):357-363.

18. Khalifa AS, Mitchell BS, Watts DM, El-Samahy MH, El-Sayed MH, Hassan NF, Jennings GB, Hibbs RG, Corwin AL: Prevalence of hepatitis C viral antibody in transfused and nontransfused Egyptian children. Am J Trop Med Hyg 1993, 49(3):316-321

19. el-Nanawy AA, el Azzouni OF, Soliman AT, Amer AE, Demian RS, el-Sayed HM: Prevalence of hepatitis-C antibody seropositivity in healthy Egyptian children and four high risk groups. J Trop Pediatr 1995, 41(6):341-343.

20. Miras A, Morris K, Soper C: Hepatitis C virus prevalence in children in a highly endemic region of Egypt. Pediatr Infect Dis J 2002, 21(10):987.

21. El-Raziky MS, El-Hawary M, Esmat G, Abouzied AM, El-Koofy N, Mohsen N, Mansour S, Shaheen A, Abdel Hamid M, El-Karaksy H: Prevalence and risk factors of asymptomatic hepatitis $C$ virus infection in Egyptian children. World I Gastroenterol 2007, 13(12):1828-1832.

22. Kandil ME, Rasheed MA, Saad NE: Hepatitis C and B viruses among some high risk groups of Egyptian children. J Med Sci 2007, 7(8):1259-1267.

23. Hassan NF, Kotkat A: Prevalence of antibodies to hepatitis $C$ virus in pregnant women in Egypt. J Infect Dis 1993, 168(1):248-249.

24. Agha S, Sherif $L S$, Allam MA, Fawzy M: Transplacental transmission of hepatitis C virus in HIV-negative mothers. Res Virol 1998, 149(4):229-234.
25. Kassem AS, El-Nawawy AA, Massoud MN, Abou El-Nazar SY, Sobhi EM: Prevalence of hepatitis $C$ virus (HCV) infection and its vertical transmission in Egyptian pregnant women and their newborns. J Trop Pediatr 2000, 46(4):231-233.

26. Stoszek SK, Abdel-Hamid M, Narooz S, El Daly M, Saleh DA, Mikhail N, Kassem E, Hawash Y, El Kafrawy S, Said A, et al: Prevalence of and risk factors for hepatitis $C$ in rural pregnant Egyptian women. Trans $R$ SoC Trop Med Hyg 2006, 100(2):102-107.

27. Zahran KM, Badary MS, Agban MN, Abdel Aziz NH: Pattern of hepatitis virus infection among pregnant women and their newborns at the Women's Health Center of Assiut University, Upper Egypt. Int J Gynaecol Obstet 2010, 111(2):171-174.

28. AbdulQawi K, Youssef A, Metwally MA, Ragih I, AbdulHamid M, Shaheen A: Prospective study of prevalence and risk factors for hepatitis $\mathrm{C}$ in pregnant Egyptian women and its transmission to their infants. Croat Med J 2010, 51(3):219-228.

29. Abo Elmagd EK, Abdel-Wahab KS, Alrasheedy ZE, Khalifa AS: An Egyptian study of mother to child transmission of hepatitis C virus. Int J Virology 2011, 7(3):100-108

30. Kamel MA, Ghaffar YA, Wasef MA, Wright M, Clark LC, Miller FD: High HCV prevalence in Egyptian blood donors. Lancet 1992, 340(8816):427.

31. el-Zayadi A, Selim O, Rafik M, el-Haddad S: Prevalence of hepatitis C virus among non-A, non-B-related chronic liver disease in Egypt. $J$ Hepatol 1992, 14(2-3):416-417.

32. Darwish NM, Abbas MO, Abdelfattah FM, Darwish MA: Hepatitis C virus infection in blood donors in Egypt. J Egypt Public Health Assoc 1992, 67(3-4):223-236

33. Darwish MA, Raouf TA, Rushdy P, Constantine NT, Rao MR, Edelman R: Risk factors associated with a high seroprevalence of hepatitis $C$ virus infection in Egyptian blood donors. Am J Trop Med Hyg 1993, 49(4):440-447.

34. Quinti I, Renganathan E, El Ghazzawi E, Divizia M, Sawaf G, Awad S, Pana A, Rocchi G: Seroprevalence of HIV and HCV infections in Alexandria, Egypt. Zentralblatt fur Bakteriologie: international journal of medical microbiology 1995, 283(2):239-244.

35. Bassily S, Hyams KC, Fouad RA, Samaan MD, Hibbs RG: A high risk of hepatitis $C$ infection among Egyptian blood donors: the role of parenteral drug abuse. Am J Trop Med Hyg 1995, 52(6):503-505.

36. Khattab MA, Eslam M, Sharwae MA, Hamdy L: Seroprevalence of hepatitis C and B among blood donors in Egypt: Minya Governorate, 2000-2008. Am J Infect Control 2010, 38(8):640-641.

37. Attia MAM, Zekri ARN, Goudsmit J, Boom R, Khaled HM, Mansour MT, De Wolf F, Alam El-Din HM, Sol CJA: Diverse patterns of recognition of hepatitis $C$ virus core and nonstructural antigens by antibodies present in Egyptian cancer patients and blood donors. J Clin Microbiol 1996, 34(11):2665-2669.

38. Arthur RR, Hassan NF, Abdallah MY, El-Sharkawy MS, Saad MD, Hackbart BG, Imam IZ: Hepatitis $\mathrm{C}$ antibody prevalence in blood donors in different governorates in Egypt. Trans R Soc Trop Med Hyg 1997, 91(3):271-274.

39. el-Zayadi AR, Selim O, Ibrahim EH, Hamdy H, Dabbous H, Ahdy A, Moneim SA: Does schistosomiasis play a role in the high sero prevalence of HCV antibody among Egyptians? Trop Gastroenterol 1997, 18(3):98-100.

40. Gad A, Tanaka E, Orii K, Rokuhara A, Nooman Z, Serwah AH, Shoair M, Yoshizawa K, Kiyosawa K: Relationship between hepatitis $C$ virus infection and schistosomal liver disease: not simply an additive effect. J Gastroenterol 2001, 36(11):753-758.

41. Tanaka Y, Agha S, Saudy N, Kurbanov F, Orito E, Kato T, Abo-Zeid M, Khalaf M, Miyakawa Y, Mizokami M: Exponential spread of hepatitis $C$ virus genotype 4a in Egypt. J Mol Evol 2004, 58(2):191-195.

42. Hashish MH, El-Barrawy MA, Mahmoud OA, Abdel Rahman NW: $\Pi$ virus among blood donors in Alexandria. J Egypt Public Health Assoc 2005, 80(5-6):651-664

43. El-Gilany AH, El-Fedawy S: Bloodborne infections among student voluntary blood donors in Mansoura University, Egypt. East Mediterr Health J 2006, 12(6):742-748.

44. Agha S, El-Mashad N, El-Malky M, El-Shony H, El-Sherif MZ, El-Hasan MA, Tanaka Y, Mizokami M: Prevalence of low positive anti-HCV antibodies in blood donors: Schistosoma mansoni co-infection and possible role of autoantibodies. Microbiol Immunol 2006, 50(6):447-452.

45. El Damaty SI, Hassan SK, Mohamed MK, El Hosini M, Rekacewicz C, Fontanet A: Surveillance system for HCV infection: testing a model based on blood banks. J Egypt Public Health Assoc 2007, 82(5-6):451-471. 
46. El-Zayadi AR, Ibrahim EH, Badran HM, Saeid A, Moneib NA, Shemis MA, Abdel-Sattar RM, Ahmady AM, El-Nakeeb A: Anti-HBc screening in Egyptian blood donors reduces the risk of hepatitis $B$ virus transmission. Transfus Med 2008, 18(1):55-61.

47. Ismail AM, Ziada HN, Sheashaa HA, Shehab El-Din AB: Decline of viral hepatitis prevalence among asymptomatic Egyptian blood donors: a glimmer of hope. Eur J Intern Med 2009, 20(5):490-493.

48. Ashour D, Moftah F, Gobran H, Ekram D: Decreasing the risk of transfusion transmitted infections (TTIS) in the Egyptian Blood Transfusion Services. Vox Sang 2009, 96:103-104.

49. Radwan D, Moftah F, Goubran H, Mosa M, Ibrahem G: Over all representation of NAT results and correlation of NAT negativity with seropositive blood donors. Vox Sang 2010, 99:87.

50. Wasfi OA, Sadek NA: Prevalence of hepatitis B surface antigen and hepatitis $C$ virus antibodies among blood donors in Alexandria, Egypt. East Mediterr Health J 2011, 17(3):238-242.

51. Awadalla HI, Ragab MH, Nassar NA, Osman MAH: Risk factors of hepatitis C infection among Egyptian blood donors. Cent Eur J Public Health 2011, 19(4):217-221

52. Farawela H, Khorshied M, Shaheen I, Gouda H, Nasef A, Abulata N, Mahmoud HA, Zawam HM, Mousa SM: The association between hepatitis $C$ virus infection, genetic polymorphisms of oxidative stress genes and B-cell non-Hodgkin's lymphoma risk in Egypt. Infect Genet Evol 2012, 12(6):1189-1194

53. Abdel-Wahab MF, Zakaria S, Kamel M, Abdel-Khaliq MK, Mabrouk MA, Salama H, Esmat G, Thomas DL, Strickland GT: High seroprevalence of hepatitis $\mathrm{C}$ infection among risk groups in Egypt. Am J Trop Med Hyg 1994, 51(5):563-567.

54. Kamel MA, Miller FD, el Masry AG, Zakaria S, Khattab M, Essmat G, Ghaffar YA: The epidemiology of Schistosoma mansoni, hepatitis $B$ and hepatitis C infection in Egypt. Ann Trop Med Parasitol 1994, 88(5):501-509.

55. Darwish NM, Abbas MO, Hady SI, Mohammed TA: Study of the high prevalence of HCV in Egypt. J Egypt Public Health Assoc 1995, 70(3-4):397-414.

56. Darwish MA, Faris $R$, Clemens JD, Rao MR, Edelman R: High seroprevalence of hepatitis $A, B, C$, and $E$ viruses in residents in an Egyptian village in the Nile delta: A pilot study. Am J Trop Med Hyg 1996, 54(6):554-558.

57. el-Sayed HF, Abaza SM, Mehanna S, Winch PJ: The prevalence of hepatitis $B$ and $C$ infections among immigrants to a newly reclaimed area endemic for Schistosoma mansoni in Sinai, Egypt. Acta Trop 1997 68(2):229-237.

58. Nafeh MA, Medhat A, Shehata M, Mikhail NNH, Swifee $Y$, Abdel-Hamid M, Watts S, Fix AD, Strickland GT, Anwar W, et al: Hepatitis C in a community in upper Egypt: I. Cross-sectional survey. Am J Trop Med Hyg 2000 63(5-6):236-241.

59. Abdel-Aziz F, Habib M, Mohamed MK, Abdel-Hamid M, Gamil F, Madkour S, Mikhail NN, Thomas D, Fix AD, Strickland GT, et al: Hepatitis C virus (HCV) infection in a community in the Nile Delta: Population description and HCV prevalence. Hepatology 2000, 32(1):111-115.

60. Darwish MA, Faris R, Darwish N, Shouman A, Gadallah M, El-Sharkawy MS, Edelman R, Grumbach K, Rao MR, Clemens JD: Hepatitis C and cirrhotic liver disease in the Nile delta of Egypt: A community-based study. Am J Trop Med Hyg 2001, 64(3-4):147-153.

61. el-Sadawy M, Ragab H, el-Toukhy H, el-Mor Ael L, Mangoud AM, Eissa MH, Afefy AF, el-Shorbagy E, Ibrahem IA, Mahrous S, et al: Hepatitis C virus infection at Sharkia Governorate, Egypt: seroprevalence and associated risk factors. J Egypt Soc Parasitol 2004, 34(1):367-384.

62. Arafa N, El Hoseiny M, Rekacewicz C, Bakr I, El-Kafrawy S, El Daly M, Aoun S, Marzouk D, Mohamed MK, Fontanet A: Changing pattern of hepatitis C virus spread in rural areas of Egypt. J Hepatol 2005, 43(3):418-424.

63. Mohamed MK, Bakr I, El-Hoseiny M, Arafa N, Hassan A, Ismail S, Anwar M, Attala M, Rekacewicz C, Zalata K, et al: HCV-related morbidity in a rural community of Egypt. J Med Virol 2006, 78(9):1185-1189.

64. Eassa S, Eissa M, Sharaf SM, Ibrahim MH, Hassanein OM: Prevalence of hepatitis $C$ virus infection and evaluation of a health education program in el-ghar village in zagazig, egypt. J Egypt Public Health Assoc 2007, 82(5-6):379-404

65. Aguilar CE, Soliman AS, McConnell DS, Zekri AR, Banerjee M, Omar A, Sharawy M, Omar S, Raouf A, Sowers MR: Androgen profiles among Egyptian adults considering liver status. J Gastroenterol Hepatol 2008, 23(7 Pt 2):e137-e145.
66. El-Sherbini A, Hassan W, Abdel-Hamid M, Naeim A: Natural history of hepatitis $C$ virus among apparently normal schoolchildren: Follow-up after 7 years. J Trop Pediatr 2003, 49(6):384-385.

67. Mohamed MK, Magder LS, Abdel-Hamid M, El-Daly M, Mikhail NN, Abdel-Aziz F, Medhat A, Thiers $V$, Strickland GT: Transmission of hepatitis $C$ virus between parents and children. Am J Trop Med Hyg 2006, 75(1):16-20.

68. El Sherbini A, Mohsen SA, Hasan W, Mostafa S, El Gohary K, Moneib A, Abaza $\mathrm{AH}$ : The peak impact of an Egyptain outbreak of hepatitis $\mathrm{C}$ virus: Has it passed or has not yet occurred? Liver Int 2007, 27(6):876-877.

69. Barakat SH, El-Bashir N: Hepatitis C virus infection among healthy Egyptian children: Prevalence and risk factors. J Viral Hepat 2011, 18(11):779-784.

70. El-Sayed NM, Gomatos PJ, Rodier GR, Wierzba TF, Darwish A, Khashaba S, Arthur RR: Seroprevalence survey of Egyptian tourism workers for hepatitis B virus, hepatitis $C$ virus, human immunodeficiency virus, and Treponema pallidum infections: Association of hepatitis $C$ virus infections with specific regions of Egypt. Am J Trop Med Hyg 1996, 55(2):179-184

71. Mohamed MK, Hussein MH, Massoud AA, Rakhaa MM, Shoeir S, Aoun AA, Aboul Naser M: Study of the risk factors for viral hepatitis $C$ infection among Egyptians applying for work abroad. J Egypt Public Health Assoc 1996, 71(1-2):113-147.

72. Gohar SA, Khalil RY, Elaish NM, Khedr EM, Ahmed MS: Prevalence of antibodies to hepatitis $C$ virus in hemodialysis patients and renal transplant recipients. J Egypt Public Health Assoc 1995, 70(5-6):465-484.

73. Halim AB, Garry RF, Dash S, Gerber MA: Effect of schistosomiasis and hepatitis on liver disease. Am J Trop Med Hyg 1999, 60(6):915-920.

74. Hassan MM, Zaghloul AS, El-Serag HB, Soliman O, Patt YZ, Chappell CL, Beasley RP, Hwang LY: The role of hepatitis $C$ in hepatocellular carcinoma: a case control study among Egyptian patients. J Clin Gastroenterol 2001, 33(2):123-126.

75. Strickland GT, Elhefni H, Salman T, Waked I, Abdel-Hamid M, Mikhail NN, Esmat G, Fix A: Role of hepatitis $C$ infection in chronic liver disease in Egypt. Am J Trop Med Hyg 2002, 67(4):436-442.

76. El-Sayed GM, Mohamed WS, Nouh MA, Moneer MM, El-Mahallawy HA: Vira genomes and antigen detection of hepatitis $B$ and $C$ viruses in involved lymph nodes of Egyptian non-Hodgkin's lymphoma patients. Egypt J Immunol 2006, 13(1):105-114.

77. El Bassuoni MA, Obada MA, Korah T, El Sayed S: Assessment of Treg cells CD4 + CD25+ in chronic cirrhotic liver disease and hepatocellular carcinoma Egyptian patients. Hepatitis Monthly 2008, 8(3):173-177.

78. Salama KM, Selim Oel S: Hepatitis G virus infection in multitransfused Egyptian children. Pediatr Hematol Oncol 2009, 26(4):232-239.

79. Farghaly $A G$, Barakat RM: Prevalence, impact and risk factors of hepatitis C infection. J Egypt Public Health Assoc 1993, 68(1-2):63-79.

80. Mohamed MK: Epidemiology of HCV in Egypt 2004. The Afro-Arab Liver Journal 2004, 3(2):41-52.

81. Sharaf-Eldeen S, Salama K, Eldemerdash S, Hassan HMS, Semesem M: Hepatitis B and C Viruses in Egyptian children with malignancy. J Med Sci 2007, 7(6):1003-1008.

82. Habib M, Mohamed MK, Abdel-Aziz F, Magder LS, Abdel-Hamid M, Gamil F, Madkour S, Mikhail NN, Anwar W, Strickland GT, et al: Hepatitis C virus infection in a community in the Nile Delta: Risk factors for seropositivity. Hepatology 2001, 33(1):248-253

83. El-Gohary A, Hassan A, Uchida T, Shikata T, Nooman Z: Prevalence of hepatitis E virus among acute sporadic hepatitis patients in Suez. Int Hepatol Commun 1994, 2(4):218-222.

84. El Gaafary MM, Rekacewicz C, Abdel-Rahman AG, Allam MF, El Hosseiny M, Hamid MA, Colombani F, Sultan Y, El-Aidy S, Fontanet A, et al: Surveillance of acute hepatitis C in Cairo, Egypt. J Med Virol 2005, 76(4):520-525.

85. Meky FA, Stoszek SK, Abdel-Hamid M, Selim S, Abdel-Wahab A, Mikhail N, ElKafrawy S, El-Daly M, Abdel-Aziz F, Sharaf S, et al: Active surveillance for acute viral hepatitis in rural villages in the Nile Delta. Clin Infect Dis 2006, 42(5):628-633.

86. Zakaria S, Fouad R, Shaker O, Zaki S, Hashem A, El-Kamary SS, Esmat G: Changing patterns of acute viral hepatitis at a major urban referral center in Egypt. Clin Infect Dis 2007, 44(4):e30-e36.

87. Kalil KA, Farghally HS, Hassanein KM, Abd-Elsayed AA, Hassanein FE: Hepatitis $C$ virus infection among paediatric patients attending University of Assiut Hospital, Egypt. Eastern Mediterranean health journal = La revue de sante de la Mediterranee orientale = al-Majallah al-sihhivah li-shara al-mutawassit 2010, 16(4):356-361 
88. Talaat M, El-Sayed N, Kandeel A, Azab MA, Afifi S, Youssef FG, Ismael T, Hajjeh R, Mahoney FJ: Sentinel surveillance for patients with acute hepatitis in Egypt, 2001-04. East Mediterr Health J 2010, 16(2):134-140.

89. Eldin SS, Seddik I, Daef EA, Shata MT, Raafat M, Abdel Baky L, Nafeh MA: Risk factors and immune response to hepatitis $E$ viral infection among acute hepatitis patients in Assiut, Egypt. Egypt J Immunol 2010, 17(1):73-86

90. Badawy AA, Khalil $\mathrm{HH}$ : The aetiology of acute viral hepatitis among some Egyptian youth. J Egypt Soc Parasitol 2012, 42(1):203-206.

91. Said ZN, El-Sayed MH, El-Bishbishi IA, El-Fouhil DF, Abdel-Rheem SE, El-Abedin MZ, Salama II: High prevalence of occult hepatitis B in hepatitis C-infected Egyptian children with haematological disorders and malignancies. Liver Int 2009, 29(4):518-524.

92. Tonbary YA, Elashry R, Zaki Mel S: Descriptive epidemiology of hemophilia and other coagulation disorders in mansoura, egypt: retrospective analysis. Mediterranean journal of hematology and infectious diseases 2010, 2(3):e2010025

93. El-Faramawy AAM, El-Rashidy OF, Tawfik PH, Hussein GH: Transfusion transmitted hepatitis: Where do we stand now? A one center study in upper Egypt. Hepatitis Monthly 2012, 12(4):286-291.

94. Abdelwahab MS, El-Raziky MS, Kaddah NA, Abou-Elew HH: Prevalence of hepatitis $C$ virus infection and human immunodeficiency virus in a cohort of Egyptian hemophiliac children. Ann Saudi Med 2012, 32(2):200-202

95. Khalifa AS, Salem M, Mounir E, El-Tawil MM, El-Sawy M, Abd Al-Aziz MM: Abnormal glucose tolerance in Egyptian beta-thalassemic patients: possible association with genotyping. Pediatr Diabetes 2004, 5(3):126-132.

96. Abdalla NM, Galal A, Fatouh AA, Eid K, Salama EEE, Gomma HE: Transfusion transmitted virus (TTV) infection in polytransfused Egyptian thalassemic children. J Med Sci 2006, 6(5):833-837.

97. Omar N, Salama K, Adolf S, El-Saeed GS, Abdel Ghaffar N, Ezzat N: Major risk of blood transfusion in hemolytic anemia patients. Blood Coagul Fibrinolysis 2011, 22(4):280-284.

98. Mansour AK, Aly RM, Abdelrazek SY, Elghannam DM, Abdelaziz SM, Shahine DA, Elmenshawy NM, Darwish AM: Prevalence of HBV and HCV infection among multi-transfused Egyptian thalassemic patients. Hematol Oncol Stem Cell Ther 2012, 5(1):54-59.

99. Bassily S, Hyams KC, El-Masry NA, Hassan NF, Watts DM: Hepatitis C virus infection and hepatosplenic schistosomiasis [1]. Scand J Infect Dis 1992, 24(5):687-688

100. Zekri ARN, Sedkey L, El-Din HMA, Abdel-Aziz AO, Viazov S: The pattern of transmission transfusion virus infection in Egyptian patients [6]. Int J Infect Dis 2002, 6(4):329-331.

101. El-Sabah AA, El-Metwally MT, Abozinadah NY: Hepatitis C and B virus in schistosomiasis patients on oral or parenteral treatment. J Egypt Soc Parasitol 2011, 41(2):307-314.

102. Hassan NF: Prevalence of hepatitis $C$ antibodies in patient groups in Egypt. Trans R Soc Trop Med Hyg 1993, 87(6):638

103. Hassan AA, Khalil R: Hepatitis $C$ in dialysis patients in egypt: relationship to dialysis duration, blood transfusion, and liver disease. Saudi J Kidney Dis Transp/ 2000, 11(1):72-73.

104. Shatat HZ, Kotkat AM, Farghaly AG: Immune response to hepatitis B vaccine in haemodialysis patients. J Egypt Public Health Assoc 2000, 75(3-4):257-275.

105. Gad A, Tanaka E, Orii K, Rokuhara A, Nooman Z, El-Hamid Serwah A, ElSherif A, El-Essawy M, Yoshizawa K, Kiyosawa K: Clinical significance of TT virus infection in maintenance hemodialysis patients of an endemic area for hepatitis C infection. Hepatology Research 2002, 22(1):13-19.

106. El Yazeed SA NAEL-G, Younes K, El-Ghobary A: Antiphospholipid antibodies in Egyptian patients with chronic renal failure. J Med Sci 2006, 6(3):468-473.

107. Attia EA, Hassan SI, Youssef NM: Cutaneous disorders in uremic patients on hemodialysis: an Egyptian case-controlled study. Int J Dermatol 2010, 49(9):1024-1030

108. Ibrahim S: Quality of care assessment and adherence to the international guidelines considering dialysis, water treatment, and protection against transmission of infections in university hospital-based dialysis units in Cairo, Egypt. Hemodial Int 2010, 14(1):61-67.

109. el-Ghazzawi E, Drew L, Hamdy L, El-Sherbini E, Sadek Sel D, Saleh E: Intravenous drug addicts: a high risk group for infection with human immunodeficiency virus, hepatitis viruses, cytomegalo virus and bacterial infections in Alexandria Egypt. J Egypt Public Health Assoc 1995, 70(1-2):127-150
110. El-Karaksy H, Anwar GH, El-Raziky MS, El-Hawary M, Hashem M, El-Sayed R, El-Shabrawi M, Mohsen N, Fouad H, Esmat G: Anti-HCV prevalence among diabetic and non-diabetic Egyptian children. Curr Diabetes Rev 2010, 6(6):388-392.

111. Elmagd MM, Bakr MA, Metwally AH, Wahab AM: Clinicoepidemiologic study of posttransplant diabetes after living-donor renal transplant. Exp Clin Transplant 2008, 6(1):42-47.

112. El-Medany OM: El-Din Abdel Wahab KS, Abu Shady EA, Gad El-Hak N: Chronic liver disease and hepatitis $C$ virus in Egyptian patients. Hepatogastroenterology 1999, 46(27):1895-1903.

113. Farghaly AG, Mansour GA, Mahdy NH, Yousri A: Hepatitis $B$ and $C$ virus infections among patients with gingivitis and adult periodontitis: seroprevalence and public health importance. J Egypt Public Health Assoc 1998, 73(5-6):707-735.

114. Morad WS: Transmission of hepatitis $C$ between spouses an epidemiological study at National Liver Institute hospital. Int J Infect Dis 2011, 15:S81.

115. El-Zayadi A, Khalifa AA, El-Misiery A, Naser AM, Dabbous H, Aboul-Ezz AA Evaluation of risk factors for intrafamilial transmission of HCV infection in Egypt. J Egypt Public Health Assoc 1997, 72(1-2):33-51.

116. Shalaby S, Kabbash IA, El Saleet G, Mansour N, Omar A, El Nawawy A: Hepatitis $B$ and $C$ viral infection: prevalence, knowledge, attitude and practice among barbers and clients in Gharbia governorate, Egypt. East Mediterr Health J 2010, 16(1):10-17.

117. Ezzat S, Abdel-Hamid M, Abdel-Latif Eissa S, Mokhtar N, Labib AN, El-Ghorory L, Mikhail NN, Abdel-Hamid A, Hifnawy T, Strickland GT, et al: Associations of pesticides, HCV, HBV, and hepatocellular carcinoma in Egypt. Int J Hyg Environ Health 2005, 208(5):329-339.

118. Meir H, Balawi I, Nayel H, El Karaksy H, El Haddad A: Hepatic dysfunction in children with acute lymphoblastic leukemia in remission: Relation to hepatitis infection. Med Pediatr Oncol 2001, 36(4):469-473.

119. Mostafa EE A, Mansour T, Amin M, Sidhom I, Khairy A, El Zomor H: Seroprevalence of Hepatitis B and C in Pediatric Malignancies. J Egypt Natl Canc Inst 2003, 15(1):33-42.

120. Medhat A, Shehata M, Magder LS, Mikhail N, Abdel-Baki L, Nafeh M, AbdelHamid M, Strickland GT, Fix AD: Hepatitis c in a community in Upper Egypt: risk factors for infection. Am J Trop Med Hyg 2002, 66(5):633-638.

121. Plancoulaine S, Mohamed MK, Arafa N, Bakr I, Rekacewicz C, Tregouet DA, Obach D, El Daly M, Thiers V, Feray C, et al: Dissection of familial correlations in hepatitis $C$ virus (HCV) seroprevalence suggests intrafamilial viral transmission and genetic predisposition to infection. Gut 2008, 57(9):1268-1274

122. Wasley A, Alter MJ: Epidemiology of hepatitis C: Geographic differences and temporal trends. Semin Liver Dis 2000, 20(1):1-16.

123. Sievert W, Altraif I, Razavi HA, Abdo A, Ahmed EA, Alomair A, Amarapurkar D, Chen $\mathrm{CH}$, Dou X, El Khayat $\mathrm{H}$, et al: A systematic review of hepatitis $\mathrm{C}$ virus epidemiology in Asia, Australia and Egypt. Liver Int 2011, 31(SUPPL. 2):61-80.

124. Aceijas C, Rhodes T: Global estimates of prevalence of HCV infection among injecting drug users. Int J Drug Policy 2007, 18(5):352-358.

125. Aceijas C, Friedman SR, Cooper HLF: Estimates of injecting drug users at the national and local level in developing and transitional countries, and gender and age distribution (vol 82, pg 10, 2006). Sex Transm Infect 2006, 82(4):344-344

126. Yahia M: Global health: A uniquely Egyptian epidemic. Nature 2011, 474(7350 SUPPL):S12-S13.

127. Breban R, Doss W, Esmat G, Elsayed M, Hellard M, Ayscue P, Albert M, Fontanet A, Mohamed M: Towards realistic estimates of HCV incidence in Egypt. J Viral Hepat 2012. doi:10.1111/j.1365-2893.2012.01650.x.

128. Waked IA, Saleh SM, Moustafa MS, Raouf AA, Thomas DL, Strickland GT: High prevalence of hepatitis $C$ in Egyptian patients with chronic liver disease. Gut 1995, 37(1):105-107.

129. Demian AD: Prevalence of anaesthetic co-morbid factors among urological patients in a tertiary referral centre in Egypt. Egyptian $\mathrm{J}$ Anaesth 2004, 20(3):325-330.

130. Madwar MA, El-Gindy I, Fahmy HM, Shoeb NM, Massoud BA: Hepatitis C virus transmission in family members of Egyptian patients with $\mathrm{HCV}$ related chronic liver disease. J Egypt Public Health Assoc 1999, 74(3-4):313-332.

131. Shebl FM, El-Kamary SS, Saleh DA, Abdel-Hamid M, Mikhail N, Allam A, El-Arabi H, Elhenawy I, El-Kafrawy S, El-Daly M, et al: Prospective cohort study of mother-to-infant infection and clearance of hepatitis $C$ in rural Egyptian villages. J Med Virol 2009, 81(6):1024-1031 
132. Hammad AM, Zaghloul MH: Hepatitis G virus infection in Egyptian children with chronic renal failure (single centre study). Ann Clin Microbiol Antimicrob 2009, 8:36.

133. El Gohary A, Hassan A, Nooman Z, Lavanchy D, Mayerat C, El Ayat A, Fawaz N, Gobran F, Ahmed M, Kawano F, et al: High prevalence of hepatitis C virus among urban and rural population groups in Egypt. Acta Trop 1995, 59(2):155-161.

134. Abu-Raddad L, Akala FA, Semini I, Riedner G, Wilson D, Tawil O:

Characterizing the HIV/AIDS epidemic in the Middle East and North Africa: Time for Strategic Action. Middle East and North Africa HIV/AIDS Epidemiology Synthesis Project. World Bank/UNAIDS/WHO Publication. Washington DC: The World Bank Press; 2010.

135. Abu-Raddad L, Hilmi N, Mumtaz G, Benkirane M, Akala FA, Riedner G, Tawil O, Wilson D: Epidemiology of HIV infection in the Middle East and North Africa. AIDS 2010, 24(Suppl 2):S5-23.

136. Mumtaz GWH, Thomas S, Riome S, Setayesh H, Riedner G, Semini I, Tawil O, Akala FA, Wilson D, Abu-Raddad L: HIV epidemics are emerging among people who inject drugs in the Middle-East and North Africa: Systematic review and data synthesis. Under review 2012

137. Fadlalla FMY, Mumtaz G, Abu-Raddad L: The epidemiology of hepatitis C virus in the Arab Maghreb countries: A systematic review and meta-analysis. Under preparation 2012.

138. Miller FDA-RL: Quantifying current hepatitis C virus incidence in Egypt. Under review 2012.

139. Saeed AA, al-Admawi AM, al-Rasheed A, Fairclough D, Bacchus R, Ring C, Garson J: Hepatitis C virus infection in Egyptian volunteer blood donors in Riyadh. Lancet 1991, 338(8764):459-460.

doi:10.1186/1471-2334-13-288

Cite this article as: Mohamoud et al.: The epidemiology of hepatitis $C$ virus in Egypt: a systematic review and data synthesis. BMC Infectious Diseases 2013 13:288.

\section{Submit your next manuscript to BioMed Central and take full advantage of:}

- Convenient online submission

- Thorough peer review

- No space constraints or color figure charges

- Immediate publication on acceptance

- Inclusion in PubMed, CAS, Scopus and Google Scholar

- Research which is freely available for redistribution 